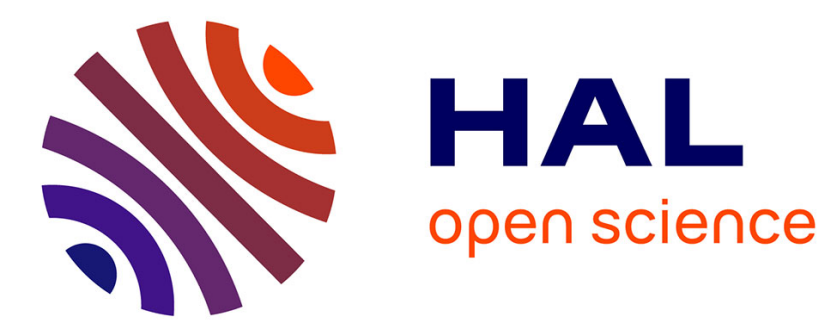

\title{
PROBABILISTIC MODEL OF BEAM-PLASMA INTERACTION IN RANDOMLY INHOMOGENEOUS PLASMA
}

A. Voshchepynets, V. Krasnoselskikh, A. Artemyev, A. Volokitin

\section{- To cite this version:}

A. Voshchepynets, V. Krasnoselskikh, A. Artemyev, A. Volokitin. PROBABILISTIC MODEL OF BEAM-PLASMA INTERACTION IN RANDOMLY INHOMOGENEOUS PLASMA. The Astrophysical Journal, 2015, 807 (1), pp.38. 10.1088/0004-637X/807/1/38 . insu-01255482

HAL Id: insu-01255482

https://hal-insu.archives-ouvertes.fr/insu-01255482

Submitted on 9 Jun 2016

HAL is a multi-disciplinary open access archive for the deposit and dissemination of scientific research documents, whether they are published or not. The documents may come from teaching and research institutions in France or abroad, or from public or private research centers.
L'archive ouverte pluridisciplinaire HAL, est destinée au dépôt et à la diffusion de documents scientifiques de niveau recherche, publiés ou non, émanant des établissements d'enseignement et de recherche français ou étrangers, des laboratoires publics ou privés. 


\title{
PROBABILISTIC MODEL OF BEAM-PLASMA INTERACTION IN RANDOMLY INHOMOGENEOUS PLASMA
}

\author{
A. Voshchepynets ${ }^{1,3}$, V. Krasnoselskikh ${ }^{1,3}$, A. Artemyev $^{2}$, and A. Volokitin $^{2}$ \\ ${ }^{1}$ LPC2E, Orleanse, France; andrii.voshchepynets@cnrs-orleans.fr \\ ${ }^{2}$ IKI RAN, Moscow, Russia \\ Received 2015 January 20; accepted 2015 March 19; published 2015 June 30
}

\begin{abstract}
We propose a new model that describes beam-plasma interaction in the presence of random density fluctuations with a known probability distribution. We use the property that, for the given frequency, the probability distribution of the density fluctuations uniquely determines the probability distribution of the phase velocity of waves. We present the system as discrete and consisting of small, equal spatial intervals with a linear density profile. This approach allows one to estimate variations in wave energy density and particle velocity, depending on the density gradient on any small spatial interval. Because the characteristic time for the evolution of the electron distribution function and the wave energy is much longer than the time required for a single wave-particle resonant interaction over a small interval, we determine the description for the relaxation process in terms of averaged quantities. We derive a system of equations, similar to the quasi-linear approximation, with the conventional velocity diffusion coefficient $D$ and the wave growth rate $\gamma$ replaced by the average in phase space, by making use of the probability distribution for phase velocities and by assuming that the interaction in each interval is independent of previous interactions. Functions $D$ and $\gamma$ are completely determined by the distribution function for the amplitudes of the fluctuations. For the Gaussian distribution of the density fluctuations, we show that the relaxation process is determined by the ratio of beam velocity to plasma thermal velocity, the dispersion of the fluctuations, and the width of the beam in the velocity space.
\end{abstract}

Key words: acceleration of particles - plasmas - solar wind

\section{INTRODUCTION}

Solar flares and their associated coronal mass ejections are the most violent examples of the acceleration of electrons within the solar system. Reconfiguration of the unstable magnetic field configuration of the solar atmosphere to lower energy states results in the acceleration of charged particles up to relativistic energies. Over a period of tens of seconds, energy up to $10^{32}$ erg can be transferred into accelerated electrons (e.g., Emslie et al. 2012).

Electron beams, ejected from the Sun during solar flares, are widely accepted to be responsible for the generation of solar type III radio bursts. These bursts are among the strongest radio emissions found within the solar system. Radio bursts have a characteristic frequency near the fundamental frequency (local electron plasma frequency) and/or its harmonics. Both the fundamental and the harmonic emission are characterized by a rapid drift, from high frequency to low, that can be assigned to the decreasing electron density of the solar wind (Lin et al. 1981). Since they can provide important information about the acceleration and transport of solar energetic electrons, as well as information regarding the conditions of the background plasma, type III radio bursts have been the subject of scientific study for the past 60 years (see the review of Reid \& Ratcliffe 2014).

Ginzburg \& Zhelezniakov (1958) were the first to propose a generation mechanism for type III radio bursts. The theory was later refined by many authors (Goldman 1983; Dulk 1985; Melrose 1987). Today, it is widely accepted that the type III radio bursts are the result of a two-step process. In the first step, electrostatic plasma waves (Langmuir waves) are generated by beam electrons. In the second step, because of scattering on plasma ions or density fluctuations, Langmuir waves can be

\footnotetext{
${ }^{3}$ LPCE/CNRS UMR7828.
}

converted to electromagnetic emission with a plasma frequency, whereas harmonic emissions appear as a result of the coalescence of two Langmuir waves. Alternative mechanisms for the conversion of the beam-driven Langmuir waves into electromagnetic radiation can be found in competing theories, including electrostatic decay (Cairns \& Melrose 1985; Cairns 1988), linear mode conversation (Hinkel-Lipsker et al. 1992), quasi-mode processes (Yoon et al. 1994), and antenna radiation (Papadopoulos \& Freund 1978; Malaspina et al. 2012).

Electron beam instability was one of the first instabilities to be described in the framework of quasi-linear (QL) theory (Drummond \& Rosenbluth 1962; Vedenov et al. 1962). QL theory indicates that beam plasma relaxation consists of several physical processes. First, an unstable beam generates Langmuir waves that grow to high enough levels to perturb the motion of particles resonant with these waves. As a result of the action of wave fields on particles, the distribution function of beam particles is modified in such a way that the wave growth rate becomes reduced. The QL approximation considers particlewave interactions only under resonant conditions. The phase velocity of the wave, $V=\omega / k$, should be equal to the particle velocity, $v$. The relaxation time due to wave-particle interaction is inversely proportional to the plasma wave energy density. The mathematical description consists of two equations. One equation describes wave growth, where the growth rate is related to the derivative of the particle distribution function. The second equation describes particle diffusion in the velocity space, suggesting the presence of a wide spectrum of waves having phase velocities in some range where the distribution function is positive during the process of evolution. Because the growth rate of the wave is proportional to the derivative of the electron velocity distribution function $f(v)$, when $f(v)$ has a positive gradient in velocity space, electrons can excite waves 
and vice versa. Waves in the region of the velocity space where $\partial f(v) / \partial v<0$ will damp. Thus, the relaxation results in plateau formation in the region where, initially, $\partial f(v) / \partial v>0$ (for more details see Vedenov \& Ryutov 1975).

From the early 1960s it has been known that QL theory, when applied to the solar corona condition, predicts a relaxation scale for the beam-plasma instability of hundreds of meters (Sturrock 1964). Because electron beams ejected from the Sun are observed at a distance of 1 AU (Buttighoffer 1998; Ergun et al. 1998; Krucker et al. 2009), such a result contradicts the observations. Some physical mechanism should be present that can decrease the level of generated Langmuir waves and suppress the beam relaxation process.

To explain the levels of wave energy density smaller than those predicted by QL theory, additional physical processes were invoked that could evacuate Langmuir waves from the velocity range resonant with particles. Such an outflow of wave energy to other regions of velocity space could be related to nonlinear processes. They could also play an important role in the evolution of generated Langmuir waves (Melrose 1980). During the 1970s, beam-plasma interaction was studied in the framework of weak (Sagdeev \& Galeev 1969; Kaplan \& Tsytovich 1973) and strong (Zakharov 1972; Galeev et al. 1977a) turbulence theory. Weak turbulence theory implies the presence of decay instability when the primary Langmuir wave decays into a secondary Langmuir wave and an ion-sound wave. The secondary wave is far from resonance with beam particles. If the process provides a rapid outflow of waves from the resonant region, the level of wave energy density in this domain significantly decreases. Due to the modulation instability, strong turbulence ensures an even more rapid outflow of wave energy from the resonant interaction region. The level of wave saturation becomes smaller and the corresponding beam relaxation length longer (Galeev et al. 1977b). However, from the estimates it follows that the length is still too short to explain the observations.

Another effect that can lead to the saturation of wave energy density on sufficiently smaller levels is related to density inhomogeneities in the plasma. Density inhomogeneities cause a change of the wave's $k$ vector and, correspondingly, the phase velocity and the amplitude for propagating waves (Kellogg et al. 1999; Bale et al. 2000). A change in phase velocity leads to a violation of the resonance condition for wave-particle interactions, which causes a change in the growth rate, $\gamma$, and a damping rate along the trajectory. Thus, it can suppress wave growth. This effect was first noticed by Breïzman \& Ruytov (1969) for a monotonically inhomogeneous plasma. Nishikawa \& Ryutov (1976) considered beam-plasma interaction in the presence of small-amplitude density fluctuations. Amplitudes of the density fluctuations were supposed to be small enough to exclude the possibility of reflection of the Langmuir waves. In this case, beam-driven Langmuir waves are scattered by density inhomogeneities without trapping. If the wave group velocity is larger than the sound velocity, this scattering can be considered elastic. The wave vector deviates from its initial direction, conserving its magnitude (for more details see Krasnoselskikh et al. 2007). The process of the interaction with each fluctuation is independent of previous interactions and results in angular diffusion of the $k$ vector. Such diffusion leads to an efficient decrease in beam-plasma interaction due to a change in the wave vector component along the velocity of the beam and to a breakup of the resonant conditions. As a result, only a portion of the wave spectrum occurs in the area of the phase space where the growth rate is positive, while, due to the diffusion, another portion is displaced to an area where waves can damp. Thus, this process leads to a decrease in the efficiency of energy transfer from beam to waves.

Ryutov (1969) proposed that when the level of fluctuations is very large, Langmuir waves generated by the beam can be trapped in density depletions. In this case, wave activity is largely localized around density minima in the form of trapped modes. However, the wave vector of the oscillations in the vicinity of the bottom of the density depletion undergoes variations from zero (in the reflection points) to some maximum value. Under such conditions, QL equations have a slightly modified form. The wave generation mechanism operating within some part of the wave's trajectory alternates with damping within the other part. The results of this study can be summarized as follows: (1) the presence of inhomogeneities results in the saturation of energy density of waves on a level significantly smaller than that for the homogeneous case, and consequently, the relaxation length of the beam becomes much longer than for the homogeneous case; and (2) due to the dispersion of the phase velocity of waves, not only to values smaller than the resonant velocity but also to larger values, the diffusion of beam particles occurs not only toward smaller velocities but also toward velocities larger than the velocity of the beam, forming a tail of accelerated particles. Numerical solutions of the equations indicate that the wave generation mechanism is switched off when the slope of the electron distribution function remains weakly positive (Voshchepynets \& Krasnoselskikh 2013).

Relaxation of the electron beam in the nonhomogeneous solar wind has been investigated by many authors in the framework of QL theory and weak turbulence theory (Kontar 2001; Li et al. 2006; Krasnoselskikh et al. 2007; Reid \& Kontar 2010; Ziebell et al. 2011). Recent numerical simulations of the beam-plasma interaction in a monotonically inhomogeneous plasma containing a negative gradient of the plasma density profile (Kontar 2001; Kontar \& Reid 2009; Reid \& Kontar 2010, 2013) indicate that electron beams ejected from the Sun lose their energy much more slowly than for the homogeneous case and can propagate over distances larger than 1 AU. Additionally, in the presence of large-scale density fluctuations, a shift of the $k$ vector for primarily generated waves to lower $k$ has been demonstrated (Reid \& Kontar 2010). Subsequent reabsorption by energetic electrons can provide quite an efficient mechanism of particle acceleration. Several recent numerical simulations for beam-plasma interactions by means of different techniques in randomly inhomogeneous plasmas (Ratcliffe et al. 2012; Krafft et al. 2013) have shown that a significant portion of the beam energy can be transferred to accelerated particles.

The first satellite observations for waveforms of Langmuir waves indicated that waves are observed in the form of intense wave packet "clumps" where the wave amplitude may reach amplitudes three orders of magnitude larger than the noise level (Gurnett et al. 1978). Smith \& Sime (1979) pointed out that the clumping phenomenon can be associated with density inhomogeneities within the solar wind. Density fluctuations can result in the rapid breakdown of the resonant conditions for the beam-plasma instability. Under such conditions, a wave along its path can pass through regions where resonant conditions for wave-particle interactions with the beam are 
satisfied and then can cross regions where these conditions are violated (Muschietti et al. 1985; Kellogg 1986; Melrose et al. 1986).

The idea that the growth rate of the beam-plasma interaction in such a plasma can behave as a random variable is the basis of the stochastic growth rate theory (SGT) proposed by Robinson (1992). He suggested that some of the most intensive waves growing up to a high level cross many regions where the growth rate of the waves is positive and should impose statistical properties of the growth rate similar to the sum of the random variables. By applying the central limit theorem, he suggested that the statistics of the increment should be normal, leading to log-normal statistics for the wave's amplitude. Lognormal statistics predicted by SGT have been reported to be observed in various contexts in natural and laboratory plasmas. These include Langmuir waves in Earth's foreshock (Cairns \& Robinson 1999) and polar cap region (Cairns \& Menietti 1999), electromagnetic ion cyclotron waves in Earth's magnetosheath (Cairns \& Grubits 2001), and eigenmodes in a laboratory vacuum arc plasma (Austin et al. 2007). However, statistical studies of the observations of Langmuir waves on board Cluster satellites indicated that deviations from the lognormal distribution are rather large (Krasnoselskikh et al. 2007). Two important effects can explain these deviations, as follows: (1) the number of regions crossed by the beam is not large enough, and (2) the saturation of waves due to waveparticle interaction can significantly change the statistical properties of wave amplitudes.

The spectra of the density fluctuations inside the solar wind were studied on various scales by making use of different techniques (Neugebauer 1975; Celnikier et al. 1983; Kellogg \& Horbury 2005; Malaspina et al. 2010; Chen et al. 2012). The spectral density of fluctuations, $W_{k}$, can be presented in the form of a double-power law. In the low-frequency (longwavelength) part of the spectrum, it can be approximated quite well using the Kolmogorov power law, as follows:

$$
W_{k} \sim\left(\frac{\Delta n}{N_{0}}\right)^{2} \sim k^{-5 / 3} .
$$

In the higher frequency range (shorter wavelengths), the power law was evaluated to be as follows:

$$
W_{k} \sim k^{-\nu}, \nu \simeq 0.64 \pm 0.01
$$

Here $N_{0}$ is the background plasma density and $\Delta n$ is the amplitude of the fluctuations. The transition occurs at a frequency of around $6 \times 10^{-2} \mathrm{~Hz}$ (Celnikier et al. 1983). Here, it is worth mentioning that in this frequency range the wave dispersion is still negligible and that the relationship between frequencies and wavelengths can be established by assuming the Taylor hypothesis. The result was obtained by Celnikier et al. (1987), making use of the technique of active sounding between two satellites. Similar results were obtained by Neugebauer (1975) using a different method. It is noteworthy that spectral indices depend on the angle between the solar wind and a background magnetic field (Forman et al. 2011). In some cases the low-frequency part of the density fluctuations spectrum is measured to be steeper than $-5 / 3$ (Celnikier et al. 1987). An important question for the beam-plasma interaction is what portion of the density fluctuation spectrum provides the most important impact on beam relaxation. Several important scales are known from studies of beam-plasma interaction in homogeneous plasmas and plasmas with monotonically changing density as compared with a density inhomogeneity scale.

Characteristic scales of the beam-plasma interaction depend on the characteristic growth rate $\gamma$. To obtain the growth of waves up to a level significantly larger than the noise level, one should satisfy the following condition:

$$
\gamma \frac{L}{v_{\mathrm{g}}} \gg \Lambda \text {. }
$$

Here $L$ is the characteristic scale of the density inhomogeneities; $v_{\mathrm{g}} \sim v_{\mathrm{t}}^{2} / v_{\mathrm{b}}$ is the group velocity of the Langmuir wave; $v_{\mathrm{t}}$ is the thermal velocity of the background plasma; and $v_{\mathrm{b}}$ is the velocity of the beam; $\Lambda$ is the so-called Coulomb logarithm, and $\Lambda=\ln N_{0} \lambda_{\mathrm{D}}^{3}$, where $\lambda_{\mathrm{D}}$ is the characteristic Debye length. If $L$ is smaller than $\left(v_{\mathrm{g}} / \gamma\right) \Lambda$, the growth of the waves is stopped on such a level that nonlinear phenomena such as waveparticle interactions can be neglected. The linear growth rate of the beam plasma instability reads

$$
\gamma \simeq \omega_{\mathrm{p}}\left(\frac{n_{\mathrm{b}}}{N_{0}}\right)\left(\frac{v_{\mathrm{b}}}{\Delta v_{\mathrm{b}}}\right)^{2},
$$

where $\omega_{\mathrm{p}}$ is the characteristic plasma frequency, $\Delta v_{\mathrm{b}}$ is the characteristic beam width in the velocity space, and $n_{\mathrm{b}}$ is the density of the beam electrons. Then the condition above can be presented as follows:

$$
L_{\min }=\left(\frac{\Delta v_{\mathrm{b}}}{v_{\mathrm{b}}}\right)^{2} \frac{N_{0}}{n_{\mathrm{b}}} \frac{v_{\mathrm{t}}}{v_{\mathrm{b}}} \Lambda \lambda_{\mathrm{D}} .
$$

For parameters relevant to solar type III electron beams and a background plasma at $1 \mathrm{AU}$, the following parameters are applicable (Ergun et al. 1998): $N_{0} \approx 5 \times 10^{6} \mathrm{~m}^{-3}$; $\omega_{\mathrm{p}} / 2 \pi \approx 20 \mathrm{kHz} ; n_{\mathrm{b}} / N_{0} \approx 10^{-5} ; \lambda_{\mathrm{D}} \approx 15 \mathrm{~m} ; \Delta v / v_{\mathrm{b}} \approx 0.05 ;$ $v_{\mathrm{b}} \approx 15 v_{\mathrm{t}}$; and $\Lambda \approx 15 ; L_{\text {min }}$ results in $250 \lambda_{\mathrm{D}}$ or $4000 \mathrm{~m}$.

Density fluctuations with very large spatial scales, larger than the beam relaxation length in a homogeneous plasma, cannot significantly influence the process of the beam-plasma interaction. Relaxation stops with a plateau formation when $\Delta v / v_{\mathrm{b}} \sim 1$. Then, $L_{\max }$ can be estimated, as follows:

$$
L_{\max }=\frac{N_{0}}{n_{\mathrm{b}}} \frac{v_{\mathrm{t}}}{v_{\mathrm{b}}} \Lambda \lambda_{\mathrm{D}} .
$$

For the parameters mentioned above, $L_{\max }$ results in $10^{5} \lambda_{\mathrm{D}}$ or $1.5 \times 10^{6} \mathrm{~m}$.

For the case of a quiet solar wind with $v_{\mathrm{SW}}=450 \mathrm{~km} \mathrm{~s}^{-1}$, $L_{\min }$ and $L_{\max }$ can yield two characteristic frequencies in the frame of spacecraft $f_{h}=120 \mathrm{~Hz}$ and $f_{l}=0.3 \mathrm{~Hz}$. The considered fluctuations correspond to a high-frequency range with a spectral index of 0.64 . Now, we consider fluctuations satisfying the following condition: $L_{\min }<L<L_{\max }$.

Two major physical effects are caused by density fluctuations, leading to changes in the wave phase velocity. Both result in changing the conditions of resonance and consequently shifting the wave-particle interaction to another part of the phase space. Thus, the wave with frequency $\omega$ and unperturbed wave vector $k_{0}$ will interact with particles that have velocities different from $\omega / k_{0}$. 
The first effect consists of the angular diffusion of the direction of wave propagation and the angular deviation of the wave vector from its supposed trajectory in a homogeneous plasma. The process becomes extremely important for density fluctuations with spatial scales, $l$, that are small compared to $\nu_{\mathrm{g}} / \gamma$, and with amplitudes that are not too large, $\left|\Delta n / N_{0}\right| \ll v_{\mathrm{t}}^{2} / v_{\mathrm{b}}^{2}$. The first of the inequalities implies that the angular deflection on each scattering event is much smaller than unity, so the process can be described by angular diffusion. The second of the inequalities states that the $k$ vector magnitude of the Langmuir waves is conserved (see Nishikawa \& Ryutov 1976 and Krasnoselskikh et al. 2007 for additional details).

The second effect consists of a change in the magnitude of $k$ in the direction of wave propagation. Density fluctuations should have amplitudes that satisfy the condition $\left|\Delta n / N_{0}\right| \geqslant v_{\mathrm{t}}^{2} / v_{\mathrm{b}}^{2}$ to be involved in this process. As shown earlier, the most important impact on beam relaxation is provided by fluctuations with spatial scales, $L$, that lie within the range $L_{\min }<L<L_{\max }$. It is worth noting that inequality (1) leads to the conclusion that $L$ is much larger than the spatial scale, $l$, of the fluctuations that are the most important for angular diffusion.

Thereby, the process of angular diffusion is dominant when density fluctuations have relatively "small" scales and amplitudes, while the effect of a change of $k$ magnitude prevails for fluctuations with larger scales and amplitudes. Our goal hereafter is to study the role of the second type of density fluctuations in beam-plasma interactions.

\section{THE MODEL}

In the following discussion, we discuss a model for beamplasma interaction in a randomly inhomogeneous plasma. We replace the continuous spatial interval with a discrete one and divide it into a set of finite, equally sized intervals of size $a$. Such discretization allows one to apply the probabilistic approach in a simpler way. The scale $a$ is stated to be much less than the characteristic scale of changes for the electron distribution function, $L_{c}$. We consider the interaction of a coherent small-amplitude wave with a particle on such a small interval by assuming that the density profile on this interval is linear. However, it is supposed that it is sufficiently larger than the wave's wavelength. Our assumptions allow one to describe the action of the field of a wave with a known frequency on a particle with a necessary degree of accuracy to calculate the effect of wave-particle interaction on any particular interval with the chosen densities at the ends. The key point of our description is that, on each interval, we assume that the values of the density in the center of the interval were random and independent and described by a predetermined, known statistical distribution. The density profile is continuous without the discontinuities being continuously skewed at the ends of neighboring intervals. The distribution can be chosen either by taking it to correspond to real observations or choosing a model distribution that allows calculations to be performed in a simpler manner. Based on this knowledge, the statistical distribution for the phase velocities of waves for any given frequency can be derived. The last distribution is uniquely determined by the distribution of the density fluctuations. The probability allows one to calculate the average energy exchange between particles with a given velocity and a wave with a given frequency. While calculating particle motion under the action of a chosen wave, we consider its phase to be determined, although, afterwards, we perform a statistical averaging over the phases by assuming that they are random and uniformly distributed in the interval from zero to $2 \pi$. We also assume that the wave-particle interaction at each interval is independent of the wave-particle interactions at previous intervals. Using such assumptions, we calculate the probability that a particle at initial time velocity $v_{0}$ after $Q$ interactions that occurs during a time interval $\left(t-t_{0}\right)$ will have a velocity $v$, where the number of steps, $Q$, should be large enough to justify statistical averaging. We replace time averaging by averaging over an ensemble with a given probability distribution. Such an assumption allows one to determine an averaging procedure over a predetermined distribution that is related to the statistical distribution of the density fluctuations, suggesting that the number of steps, $Q$, is large. An assumption of random and independent interactions corresponding to an uncorrelated Markovian process leads to a description of the evolution of the particle distribution function based on an equation of diffusion similar to the Fokker-Planck equation in velocity space. Under such assumptions, the diffusion coefficient so determined is dependent on the probability distribution of the density fluctuations. Similar to calculating the growth rate of a wave, we use the energy change of the particles and take into account the fact that on a small interval with a linear density profile, the gain or loss of energy by the particle is equal to the energy loss or gain by the wave. Local conservation of energy occurs on small intervals and allows one to calculate an average energy change for wave energy density and the increment of instability or damping for a wave having a given frequency.

Our approach consists of the exact calculation of the interaction of a coherent wave with a fixed initial phase with a particle with a given initial velocity over a small interval of size $a$. The process is deterministic and the calculations are exact. Afterward, we average the results, assuming that the intervals are random and characterized by the probability distribution function. Therefore, the averaging for waves having a known frequency was performed over random distributions of the wave's $k$ vectors (or phase velocities) and the initial phases. The sequence of such processes results in the diffusion of particles in the velocity space as described by the diffusion equation (derived subsequently). An important characteristic of this procedure is the size of the interval $a$, where the process is dynamic and deterministic. We have already noted that it should be larger than the wavelength $a \gg \lambda$. Another important limitation for $a$ can be obtained by considering the velocity change on an interval with an inhomogeneity with a linear profile: $N(x)=N_{0}+\Delta n x / L$. The dispersion relationship for the Langmuir wave can be written as follows: $\omega=\omega_{\mathrm{p}}(x)\left(1+(3 / 2)\left(v_{\mathrm{t}}^{2} / V^{2}\right)\right)$. Here $\omega$ and $V$ are the frequency and the phase velocity of the wave. Assuming that the frequency of the wave and the thermal velocity of particles are constant, the following equation can obtained:

$$
\Delta \omega=\left(1+\frac{3}{2} \frac{v_{\mathrm{t}}^{2}}{V^{2}}\right) \frac{d \omega_{\mathrm{p}}(x)}{d x} \Delta x-3 \omega_{\mathrm{p}} \frac{v_{\mathrm{t}}^{2}}{V^{2}} \frac{\Delta V}{V}=0
$$


where $\Delta V$ is the deviation of the phase velocity on the scale $\Delta x$ caused by a density gradient. Because

$$
\frac{1}{\omega_{\mathrm{p}}} \frac{d \omega_{\mathrm{p}}(x)}{d x}=\frac{1}{2} \frac{d}{d x}\left(\frac{x}{L} \frac{\Delta n}{N_{0}}\right)=\frac{1}{2 L} \frac{\Delta n}{N_{0}},
$$

and taking into account the fact that $V \sim v_{\mathrm{b}} \gg v_{\mathrm{t}}, \Delta V$ can be estimated as follows:

$$
|\Delta V| \sim v_{\mathrm{b}} \frac{\Delta x}{L} \frac{|\Delta n|}{N_{0}} \frac{v_{\mathrm{b}}}{v_{\mathrm{t}}} .
$$

Langmuir waves can grow or decay due to interaction with the electron beam, whereas the phase velocity, $V$, remains in the interval $v_{\mathrm{b}}-\Delta v_{\mathrm{b}}<V<v_{\mathrm{b}}+\Delta v_{\mathrm{b}}$. The interaction will stop when $\Delta V \sim \Delta v_{\mathrm{b}}$ and when the resonant conditions are no longer satisfied for beam particles. This occurs on the following spatial scale:

$$
\Delta x_{c}=L \frac{N_{0}}{\Delta n} \frac{\Delta v_{\mathrm{b}}}{v_{\mathrm{b}}} \frac{v_{\mathrm{t}}^{2}}{v_{\mathrm{b}}^{2}}
$$

For the condition mentioned before $\left(\Delta v_{\mathrm{b}} / v_{\mathrm{b}}=0.05\right.$, $\left.v_{\mathrm{b}}=15 v_{\mathrm{t}}\right)$ and $\Delta n / N_{0}=0.01$, waves escape from resonance on the scale $\Delta x_{c} \sim 0.02 L$. In our model, the change of velocity on one single interval should be much smaller than a velocity variation that would lead to a violation of the resonant condition. This implies that the characteristic scale for the subinterval $a$ is sufficiently smaller than $\Delta x_{c}$. In this case, a wave can be considered to remain in resonance with the electron beam on a single interval without loss of coherence.

Another effect that can lead to the violation of the resonance condition is angular diffusion. This effect is described by the diffusion equation (Nishikawa \& Ryutov 1976; Krasnoselskikh et al. 2007), and the corresponding process is characterized by a diffusion coefficient that can be estimated to be on the order of

$$
D \sim \omega_{\mathrm{p}} \frac{1}{l k} \frac{1}{\left(k^{2} \lambda_{\mathrm{D}}^{2}\right)}\left|\frac{\Delta n_{\mathrm{ad}}}{N_{0}}\right|^{2} .
$$

Here, $k$ is the characteristic wave vector of the primary Langmuir wave generated due to beam-plasma interaction, and $\Delta n_{\mathrm{ad}}$ and $l$, the amplitude and spatial scale of the inhomogeneity involved in the process of angular diffusion. To significantly reduce the instability growth, the wave vector should deviate from its initial direction to the angle $\theta$, as determined by the following condition:

$$
1-\cos \theta=\frac{\Delta v_{\mathrm{b}}}{v_{\mathrm{b}}} .
$$

If $\Delta v_{\mathrm{b}} / v_{\mathrm{b}} \ll 1$ then $\theta \sim\left(\Delta v_{\mathrm{b}} / v_{\mathrm{b}}\right)^{1 / 2}$. Thus, the characteristic time of angular diffusion in the velocity space can be estimated as follows:

$$
D \tau_{\mathrm{ad}} \sim \frac{\Delta v_{\mathrm{b}}}{v_{\mathrm{b}}},
$$

and

$$
\tau_{\mathrm{ad}} \simeq \frac{1}{D}\left(\frac{\Delta v_{\mathrm{b}}}{v_{\mathrm{b}}}\right)=\omega_{\mathrm{p}}^{-1} \frac{\left(k^{2} \lambda_{\mathrm{D}}^{2}\right)}{\left|\frac{\Delta n_{\mathrm{ad}}}{N_{0}}\right|^{2}} k l\left(\frac{\Delta v_{\mathrm{b}}}{v_{\mathrm{b}}}\right) .
$$

Now one can compare the time of the angular diffusion, $\tau_{\mathrm{ad}}$, and the time of propagation, $\tau_{\mathrm{pr}}\left(\Delta x_{c}\right)$, of a Langmuir wave through the interval $\Delta x_{c}$ :

$$
\frac{\tau_{\mathrm{ad}}}{\tau_{\mathrm{pr}}\left(\Delta x_{c}\right)}=\frac{l}{L}\left(\frac{\Delta n}{\Delta n_{\mathrm{ad}}}\right)^{2} k^{2} \lambda_{\mathrm{D}}^{2} \frac{N_{0}}{\Delta n} \frac{v_{\mathrm{b}} k}{\omega_{\mathrm{p}}}
$$

Taking into account that $v_{\mathrm{b}} k / \omega_{\mathrm{p}} \sim 1, \quad l / L \leqslant 1 / \Lambda$, $\Delta n / \Delta n_{\mathrm{ad}} \gg 1$, and $\Delta n / N_{0} \geqslant k^{2} \lambda_{\mathrm{D}}^{2}$, one can find $\tau_{\mathrm{ad}} / \tau_{\mathrm{pr}}\left(\Delta x_{c}\right) \gg 1$. Thereby, the effect of the angular diffusion is too "slow" to make a significant influence on the waveparticle interactions on the subinterval $a \ll \Delta x_{c}$. Observations of the Langmuir waves in the solar wind show that most Langmuir waves have angles with a background magnetic field smaller than $20^{\circ}$ (Ergun et al. 2008; Malaspina \& Ergun 2008; Krasnoselskikh et al. 2011), which gives an additional argument that validates the weakness of the angular diffusion of waves.

Considering density variations to be linear on scale $a$, a linear approximation can be said to be valid for an electron plasma frequency with the same degree of accuracy, causing changes in the wave phase velocity. Using the dispersion relationship for Langmuir waves, one can obtain a relationship between the plasma frequency and the corresponding phase velocity as follows: $\omega_{\mathrm{p}}^{2}(n)=\omega^{2}\left(1-3 v_{\mathrm{t}}^{2} / V^{2}\right)$, where $\omega_{\mathrm{p}}(n)$ depends on the density fluctuations.

Knowledge of the distribution function for density fluctuations at the edges of subinterval $a$ allows one to find the distribution function of the corresponding plasma frequencies, $f_{\omega_{\mathrm{p}}}\left(\omega_{\mathrm{p}}\right)$. To evaluate the probability that resonant conditions for wave-particle interactions are satisfied on any selected interval having a known density, one should calculate the common probability that the wave phase velocity is larger than the particle velocity on one end of the subinterval and smaller on the other end. The probability can be found by making use of the following expression:

$$
P_{\omega}(V)=P\left(u_{i 1}<V\right) \cdot P\left(u_{i 2}>V\right) .
$$

Here, $P\left(u_{i 1}<V\right)$ is the probability that the phase velocity $u_{i 1}$ on one end of the interval is less than the given value of the wave phase velocity, $V$, as follows: $P\left(u_{i 1}<V\right)=\int_{0}^{\omega_{\mathrm{p}}(V)}$ $f_{\omega_{\mathrm{p}}}\left(\omega_{\mathrm{p}}\right) d \omega_{\mathrm{p}}$. In a similar manner, $P\left(u_{i 2}>V\right)$ is the probability that on the other end of the subinterval the phase velocity $u_{i 2}$ is larger than $V$ and can be calculated as follows: $P\left(u_{i 2}>V\right)=\int_{\omega_{\mathrm{p}}(V)}^{\infty} f_{\omega_{\mathrm{p}}}\left(\omega_{\mathrm{p}}\right) d \omega_{\mathrm{p}}$. We consider that a change in phase velocity on an interval caused by the inhomogeneity is much less than its magnitude $\Delta V(\Delta n) / V \ll 1$. After normalizing $P_{\omega}(V)$ to one, $P_{\omega}(V) d V$ can be interpreted as the ratio of the number of subintervals $a$ on the characteristic scale $L_{c} \gg L$ to the total number of subintervals where a wave with constant frequency $\omega$ has a phase velocity $V$. 


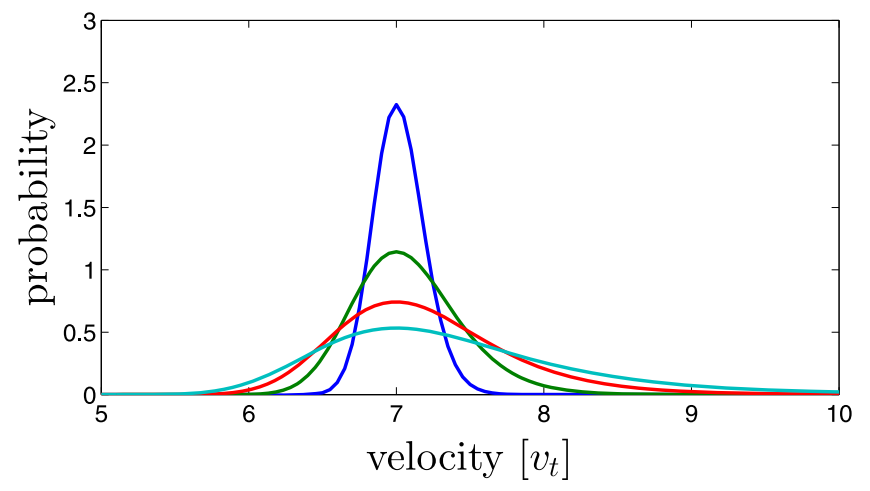

Figure 1. Examples of the probability distribution functions, $P_{\omega}(V)$, for the various fluctuation levels; $P_{\omega}(V) d V$ is the probability that a wave with a ratio of $\omega / k_{0}=7 v_{\mathrm{t}}$ will have a phase velocity $V$ at a given interval; $P_{\omega}$ was normalized to one using $\int_{0}^{\infty} P_{\omega}(V) d V=1$. The colors correspond to various fluctuation levels as follows: $\langle\delta n\rangle / N_{0}=0.005$, blue; $\langle\delta n\rangle / N_{0}=0.01$, green; $\langle\delta n\rangle / N_{0}=0.015$, red; $\langle\delta n\rangle / N_{0}=0.02$, cyan.

Another important effect to be taken into account is the probability that a wave can be reflected from a region where the plasma frequency becomes equal to the wave frequency. The result can occur one or many times. For the sake of simplicity, we calculate the total probability that the wave is reflected, including all multiple possible reflections. In terms of the probability, the probability of the single reflection can be written as follows: $P_{r}(\omega)=\int_{\omega}^{\infty} f_{\omega_{\mathrm{p}}}\left(\omega_{\mathrm{p}}\right) d \omega_{\mathrm{p}}$. The generated wave maintains its initial direction if there are no reflections at all or if the reflections compensate for one another. In the limit $L_{c} / a \rightarrow \infty$ the total probability that the wave keeps its initial direction after multiple reflections can be estimated as follows: $P_{\text {ret }}(\omega)=1-P_{r}(\omega)+P_{r}^{2}(\omega)\left(1-P_{r}(\omega)\right)+P_{r}^{4}(\omega)\left(1-P_{r}(\omega)\right)$ $+\ldots=1 /\left(1+P_{r}(\omega)\right)$. The probability that the wave moves in the opposite direction is $P_{\mathrm{opp}}(\omega)=P_{r}(\omega) /\left(1+P_{r}(\omega)\right)$.

To derive a complete description in terms of the probability distributions, an assumption should be made regarding the statistics of the density fluctuations. For the sake of simplicity, hereafter, we use the Gaussian distribution for the magnitudes of plasma frequency at the ends of subintervals $f_{\omega_{\mathrm{p}}}\left(\omega_{\mathrm{p}}\right)=\exp -\left(\omega_{\mathrm{p}}-\omega_{\mathrm{p} 0}\right)^{2} / \Omega^{2}$, where $\omega_{\mathrm{p} 0}$ is the plasma frequency of the unperturbed plasma and $\Omega$ is the dispersion of the distribution. A ratio $\Omega / \omega_{\mathrm{p} 0}$ can easily be rewritten as $(1 / 2)\left(\langle\delta n\rangle / N_{0}\right)$, where $\langle\delta n\rangle$ is the dispersion of the density fluctuations. Obviously, the probability distribution function that corresponds to the observed spectrum of density fluctuations (Celnikier et al. 1987; Kellogg \& Horbury 2005; Chen et al. 2012) is non-Gaussian. However, it seems to be reasonable to use a Gaussian distribution as a first-step approximation. A detailed study of the effects related to a deviation from Gaussian statistics is beyond the scope of the present paper. Here, it is worth mentioning that rare, largeamplitude fluctuations (that correspond to non-Gaussian "wings" of the distribution function) can be included in the proposed model because the spatial scale of the subinterval is still small enough to ensure that Langmuir waves remain in resonance with a beam on a subinterval. Figure 1 provides examples of $P_{\omega}(V)$ for a wave with a ratio of $\omega / k_{0}=7 v_{\mathrm{t}}$, where $k_{0}$ corresponds to the wave vector of the resonant Langmuir wave in a plasma without density fluctuations having an average density of $N_{0}$ for various levels of density fluctuations.
An increase in the magnitudes of the density fluctuations results in a broadening of the probability distribution function in the velocity space. Density fluctuations with larger magnitudes should make the plasma frequency stronger, leading to larger variations of the wave vector and, hence, resulting in an increase in the dispersion of the phase velocity of the wave. In the case of $\langle\Delta n\rangle / N_{0} \rightarrow 0$, the probability function $P_{\omega}(V)$ tends toward the Dirac delta function, corresponding to a homogeneous plasma where the wave can have only a fixed phase velocity, $V=\omega / k_{0}$.

\section{BASIC EQUATIONS}

We begin by considering the interaction of a singe particle and a monochromatic wave with a frequency $\omega$. Using perturbation theory, a change in the electron velocity $\Delta v$ can be estimated after passing through an interval $a$, depending on the phase difference $\phi_{\text {in }}$ between the electron and the wave in the interval beginning and on the phase velocity of the wave $V$. By assuming that $\phi_{\text {in }}$ is a random quantity with a uniform distribution and then taking $P_{\omega}(V)$ as a distribution function for the phase velocity (uniquely related to the probability distribution function of the density fluctuations), an average $\langle\Delta v\rangle_{\phi, V}$ and $\left\langle\Delta v^{2}\right\rangle_{\phi, V}$ and an average change in the particle energy, $\Delta E_{\mathrm{p}}$, after crossing the interval can be calculated. For the case of an ensemble of particles with a velocity distribution function $f(v, t)$, the change of the wave energy density, $W$, is proportional to the change in the total energy of the particles on the interval $a$ taken with opposite sign. The procedure allows averaging over many small intervals $a$ and the development of an equation for the variation of $W$ over a large interval, $L_{c}$, as follows (for details see Appendix A):

$$
\frac{d W}{d t}=\frac{\omega_{\mathrm{p} 0}}{\pi} \frac{n_{\mathrm{b}}}{N_{0}} W \int_{0}^{\infty} V^{2} \frac{\partial f}{\partial V} P_{\omega}(V) d V .
$$

Equation (3) is similar to the corresponding equation for wave energy density derived in the framework of QL theory. The difference between these equations is that in Equation (3) the wave's growth rate $\gamma$ is averaged over the phase velocity distribution. Thus, we assume that the wave can interact resonantly with particles belonging to a large interval in the velocity space because the wave phase velocity can have different values at different intervals because of density fluctuations. A similar approach was employed by Robinson (1995) in SGT. In the limit of a homogeneous plasma, $P_{\omega}(V)$ is the Dirac delta function, and Equation (3) can be written exactly as in QL theory.

We assume that the wave amplitude and the electron distribution function, $f(v, t)$, change slowly, that their variations are small on the time interval of a single waveparticle interaction, and that interactions (with neighboring intervals) are independent. Let us introduce the probability density function $U\left(v, t \mid v_{0}, t_{0}\right)$, which determines the probability that a particle with a velocity of $v_{0}$ at moment $t_{0}$ will have velocity $v$ at the moment $t$. The above-listed assumptions allow one to derive for the probability density function $U\left(v, t \mid v_{0}, t_{0}\right)$ the equation of Fokker-Planck. To obtain the equation describing the evolution of the velocity distribution function, $f(v, t), U\left(v, t \mid v_{0}, t_{0}\right)$ should be multiplied by the initial distribution $f\left(v_{0}, t_{0}\right)$ and integrated over $v_{0}$. After substitution of $\langle\Delta v\rangle_{\phi, V}$ and $\left\langle\Delta v^{2}\right\rangle_{\phi, V}$, an equation describing the evolution of an electron distribution function that has the form 
of the diffusion equation (for details see Appendix B) can be written as follows:

$$
\frac{\partial f}{\partial t}=\frac{2 e^{2} W}{m^{2} \omega} \frac{\partial}{\partial v} v P_{\omega}(v) \frac{\partial f}{\partial v} .
$$

To consider the interaction of the beam with several monochromatic waves with energy densities $W_{i}$ and frequencies $\omega_{i}$, the contributions from each wave in Equation (4) should be summed. For this case, the system of equations can be written as follows:

$$
\frac{\partial f}{\partial t}=\frac{\partial}{\partial v} v \sum_{i=1}^{K} W_{i} P_{\omega i}(v) \frac{\partial f}{\partial v},
$$

and

$$
\frac{d W_{i}}{d t}=\int_{0}^{\infty} W_{i} V^{2} \frac{\partial f}{\partial V} P_{\omega_{i}}(V) d V .
$$

To better adapt Equations (5) and (6) for the numerical simulations, it is worth introducing the dimensionless variables, as follows: $\tilde{t}=\left(\pi / \omega_{\mathrm{p} 0}\right)\left(N_{0} / n_{\mathrm{b}}\right) t, \quad \tilde{v}=v_{\mathrm{t}} v, \quad \tilde{f}=f / v_{\mathrm{t}}$, $\tilde{P}_{\omega}(V)=P_{\omega}(V) / v_{\mathrm{t}}$, and $\tilde{W}=2 \pi^{2} n_{\mathrm{b}} m v_{\mathrm{t}}^{2} W$. We omitted the tildes to simplify the form of the equations.

The system conserves the total energy, as follows:

$$
\frac{d}{d t}\left(\frac{1}{2} \int_{0}^{\infty} v^{2} f(v) d v+\sum_{i=1}^{K} W_{i}\right)=0
$$

The first term in Equation (7) corresponds to the electron energy. The second term corresponds to the total energy of the waves (for details see Appendix C).

For this study we performed numerical simulations of the system using Equations (5) and (6), and we present the results in the next section. We used a set of 2000 waves, with $\omega / k_{0}$ uniformly distributed in the range from $2 v_{\mathrm{t}}$ to $38 v_{\mathrm{t}}$. To solve the system we applied a Leapfrog method. We used Simpson's rule in order to obtain the integration in Equation (6).

\section{RESULTS}

Numerical solutions for Equations (5) and (6) for $\langle\delta n\rangle / N_{0}=0.02$ are presented in Figure 2. We use a Gaussian distribution by employing the beam velocity $v_{\mathrm{b}}=10 v_{\mathrm{t}}$ and the dispersion $\Delta v_{\mathrm{b}}=0.5 v_{\mathrm{t}}$ as the initial conditions for the electron distribution function. The case presented corresponds to the condition $(1 / 2)\left(\langle\delta n\rangle / N_{0}\right)=v_{\mathrm{t}}^{2} / v_{\mathrm{b}}^{2}$, when density fluctuations play a significant role in the relaxation process. As can be seen, beam relaxation results in plateau formation in the velocity range of $v<v_{\mathrm{b}}$, as for a homogeneous plasma. One can expect such a result because, for Langmuir waves with a large $k$ vector (and correspondingly small phase velocity) that satisfy condition $v_{\mathrm{t}}^{2} / V^{2} \gg(1 / 2)\left(\langle\delta n\rangle / N_{0}\right)$, the influence of the density fluctuations on beam relaxation is negligible. However, the number of particles with velocities $v>v_{\mathrm{b}}$ grows during the relaxation process. The energy transfer to energetic particles is possible because fluctuations of plasma density change the wave phase velocity and the resonant condition for waveparticle interactions. Thus, a wave generated with a phase velocity of $V_{0}$ can be reabsorbed by electrons with velocities $v_{e}>V_{0}$ and even $v_{e} \gg V_{0}$. The reabsorption of generated waves also leads to an increase in the relaxation time.

The left panel of Figure 3 provides the initial electron distribution function, $f_{\text {in }}(v)$, and the distribution function,

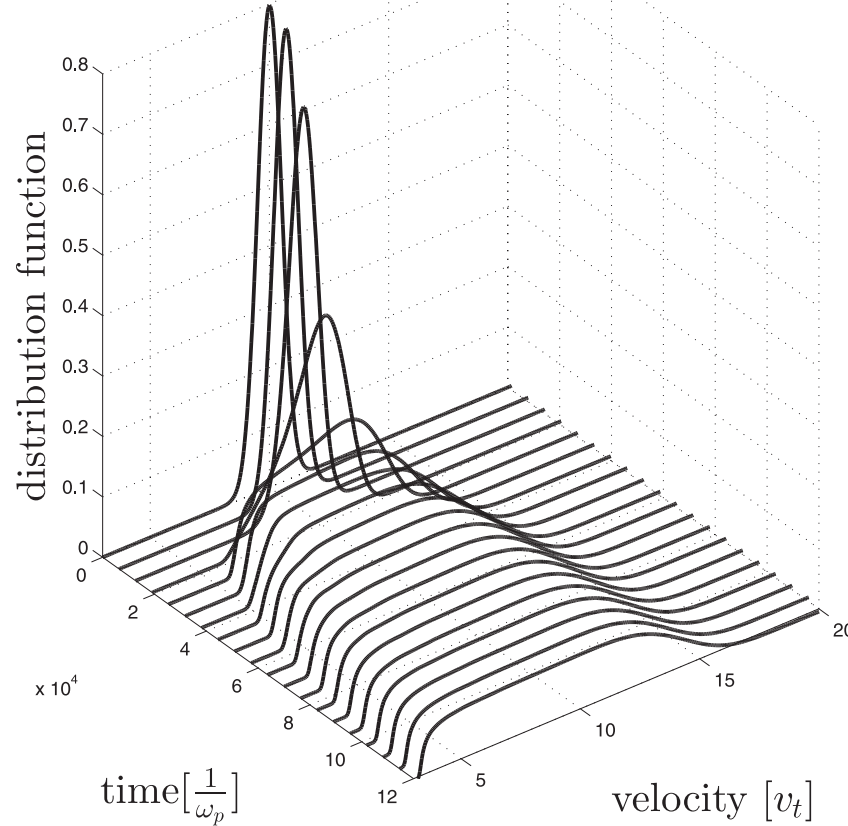

Figure 2. Evolution of the electron distribution function. The initial condition for beam electrons is the Gaussian distribution with a beam velocity of $v_{\mathrm{b}}=10 v_{\mathrm{t}}$ and a velocity dispersion of $\Delta v_{\mathrm{b}}=0.5 v_{\mathrm{t}}$. The core distribution, which corresponds to the cold electrons, has not been considered. The dimensionless electron distribution function was normalized to zero. Axes: velocities normalized to the thermal velocity of the background plasma $v_{\mathrm{t}}$; the time normalized to the backward plasma frequency $\left(1 / \omega_{\mathrm{p}}\right)$. The beam density is $n_{\mathrm{b}} / N_{0}=2.5 \times 10^{-5}$. The level of fluctuations is $\langle\delta n\rangle / N_{0}=0.02$.

$f_{\text {end }}(v)$, at the end of the relaxation process. To consider the influence of density fluctuations on the acceleration of particles, we selected particles with velocities larger than $v_{\mathrm{b}}+3 \Delta v_{\mathrm{b}}$. Thus, the number of accelerated electrons was determined as a difference between the integrals $\int_{v_{\mathrm{b}}+3 \Delta v_{\mathrm{b}}}^{\infty} f_{\text {end }}(v) d v$ and $\int_{v_{\mathrm{b}}+3 \Delta v_{\mathrm{b}}}^{\infty} f_{\text {in }}(v) d v$. In a similar manner, the energy of the accelerated particles can be found from $\frac{1}{2}\left[\int_{v_{\mathrm{b}}+3 \Delta v_{\mathrm{b}}}^{\infty} v^{2} f_{\text {end }}(v) d v-\int_{v_{\mathrm{b}}+3 \Delta v_{\mathrm{b}}}^{\infty} v^{2} f_{\text {in }}(v) d v\right]$.

The left panel of Figure 4 provides the ratio for the energy of accelerated particles, $E_{\mathrm{a}}$, to the initial energy of the beam, $E_{\mathrm{b} 0}$, for beams with different beam velocities and different levels of density fluctuations. The right panel of Figure 4 provides the relative numbers, $n_{\mathrm{a}} / n_{\mathrm{b}}$, of accelerated electrons. For instance, for the beam velocity $v_{\mathrm{b}}=6 v_{\mathrm{t}}$ and density fluctuation level 0.005 , the energy of accelerated particles is less than $0.1 \%$ from the initial energy of the beam. For the same level of fluctuations but for a beam with $v_{\mathrm{b}}=12 v_{\mathrm{t}}$ the ratio of the energy of accelerated particles increases to $29 \%$. An increase in the beam velocity leads to an inequality, $(1 / 2)\left(\langle\delta n\rangle / N_{0}\right) \gg v_{\mathrm{t}}^{2} / v_{\mathrm{b}}^{2}$, and, thus, results in a more effective acceleration of the particles. Similar results can be obtained by increasing the level of the density fluctuations. For instance, for a beam with $v_{\mathrm{b}}=8 v_{\mathrm{t}}$ the number of accelerated particles for $\langle\delta n\rangle / N_{0}=0.01$ is $7 \% n_{\mathrm{b}}$, and $30 \% n_{\mathrm{b}}$ for a case with $\langle\delta n\rangle / N_{0}=0.035$. Therefore, all cases can be separated into the following three classes: (1) $(1 / 2)\left(\langle\delta n\rangle / N_{0}\right) \ll v_{\mathrm{t}}^{2} / v_{\mathrm{b}}^{2}$, corresponding to a homogeneous plasma with fluctuations too small to influence the relaxation 

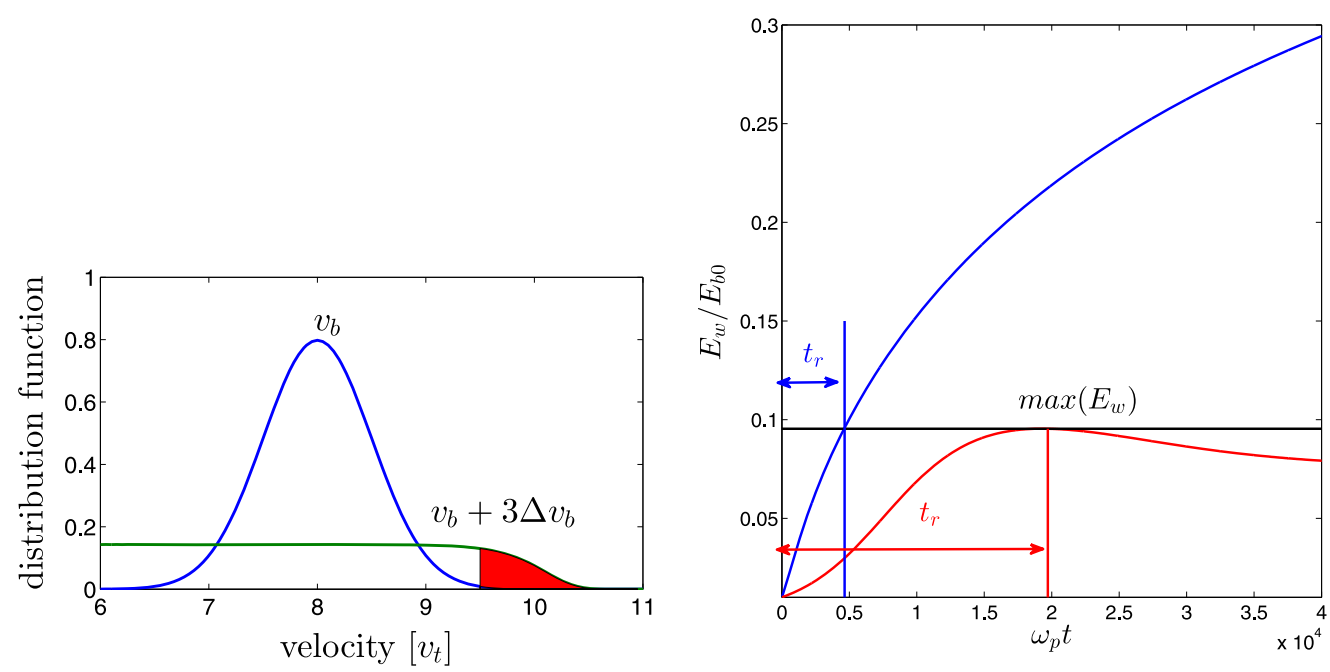

Figure 3. Left panel: the electron distribution function at the beginning of relaxation (blue line) and at the end (green line). The level of fluctuation is $1 \%$. The beam velocity is $v_{\mathrm{b}}=8 v_{\mathrm{t}}$, and the thermal velocity of the beam is $0.5 v_{\mathrm{t}} ; n_{\mathrm{b}} / N_{0}=2.5 \times 10^{-5}$. The filled area corresponds to accelerated particles. Right panel: the evolution of the total energy of waves, $E_{\mathrm{w}}$, for different levels of fluctuations $\left(\langle\delta n\rangle / N_{0}=0.005\right.$, the blue line; $\langle\delta n\rangle / N_{0}=0.04$, the red line). The beam velocity $v_{\mathrm{b}}=8 v_{\mathrm{t}}$; $\Delta v_{\mathrm{b}}=0.5 v_{\mathrm{t}} ;$ and $n_{\mathrm{b}} / N_{0}=2.5 \times 10^{-5}$.
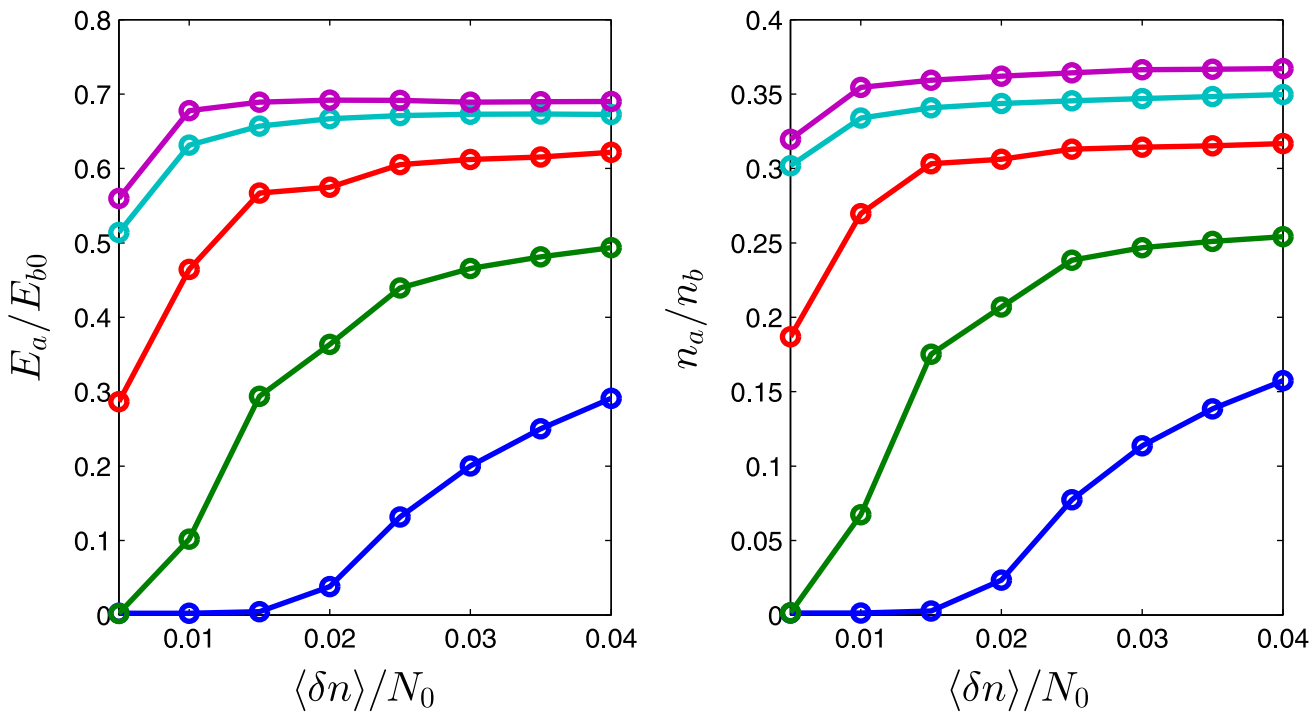

Figure 4. The energy, $E_{\mathrm{a}}$, (left panel) and the number, $n_{\mathrm{a}}$, (right panel) of accelerated particles as a function of the density fluctuation level; $E_{\mathrm{b} 0}$ and $n_{\mathrm{b}}$ are the initial energy of the beam and the density of the beam electrons. The colors correspond to the various velocities of the beam, as follows: $v_{\mathrm{b}}=6 v_{\mathrm{t}}$, blue; $v_{\mathrm{b}}=8 v_{\mathrm{t}}$, green; $v_{\mathrm{b}}=12 v_{\mathrm{t}}$, red; $v_{\mathrm{b}}=16 v_{\mathrm{t}}$, cyan; and $v_{\mathrm{b}}=20 v_{\mathrm{t}}$, magenta. The thermal velocity of all beams is $\Delta v_{\mathrm{b}}=0.5 v_{\mathrm{t}}$.

process; $(2)(1 / 2)\left(\langle\delta n\rangle / N_{0}\right) \sim v_{\mathrm{t}}^{2} / v_{\mathrm{b}}^{2}$, an intermediate regime, characterized by a presence but containing a small quantity of accelerated particles; and (3) $(1 / 2)\left(\langle\delta n\rangle / N_{0}\right) \gg v_{\mathrm{t}}^{2} / v_{\mathrm{b}}^{2}$, fluctuations resulting in an effective energy transfer to both cold and energetic particles where the energy of the accelerated electrons can reach up to $70 \%$ of the initial beam energy. Both quantities, $n_{\mathrm{a}} / n_{\mathrm{b}}$ and $E_{\mathrm{a}} / E_{\mathrm{b} 0}$, manifest the same tendency with increasing fluctuation levels. For cases of the third class, a simple relationship can be determined, as follows: $n_{\mathrm{a} /} / n_{\mathrm{b}}, E_{\mathrm{a}} / E_{\mathrm{b} 0} \sim \sqrt{\langle\delta n\rangle / N_{0}}$.

The right panel of Figure 3 provides the evolution of the total energy of waves for the case of $v_{\mathrm{b}}=8 v_{\mathrm{t}}$ and various fluctuation levels. The blue line corresponds to $\langle\delta n\rangle / N_{0}=0.005$ and for this case we have $(1 / 2)\left(\langle\delta n\rangle / N_{0}\right) \ll v_{\mathrm{t}}^{2} / v_{\mathrm{b}}^{2}$. As for a homogeneous plasma, the energy of the waves grows in time until a plateau formation is obtained. An increase in the level of density fluctuations results in a larger dispersion of the phase velocities and makes the absorption of generated waves more effective. Such can be seen in the case of $\langle\delta n\rangle / N_{0}=0.04$ (the red line shown in the right panel of Figure 3). Following a period of growth, waves begin to decay, corresponding to a phase of electron acceleration.

Ratios for the total energy of waves, $E_{\mathrm{w}}$, to the initial beam energy as a function of the fluctuations are provided in the left panel of Figure 5. Colors correspond to the various beam velocities. As one can see, in the limit of a homogeneous plasma $\left(v_{\mathrm{b}}=6 v_{\mathrm{t}}\right.$ and $\left.\langle\delta n\rangle / N_{0}=0.005\right)$, a ratio of $30 \%$ is in good agreement with the QL approximation. An increase in the magnitude of the density fluctuations leads to a decrease in the wave energy. For instance, for the same beam, but for a case of $\langle\delta n\rangle / N_{0}=0.04$, the ratio $E_{\mathrm{a}} / E_{\mathrm{b} 0}$ is two times less than for the previously mentioned case. For cases with $(1 / 2)\left(\langle\delta n\rangle / N_{0}\right) \gg v_{\mathrm{t}}^{2} / v_{\mathrm{b}}^{2}$ (the right bottom portion of the plot) the energy of the waves is less than $10 \%$ of the initial beam 

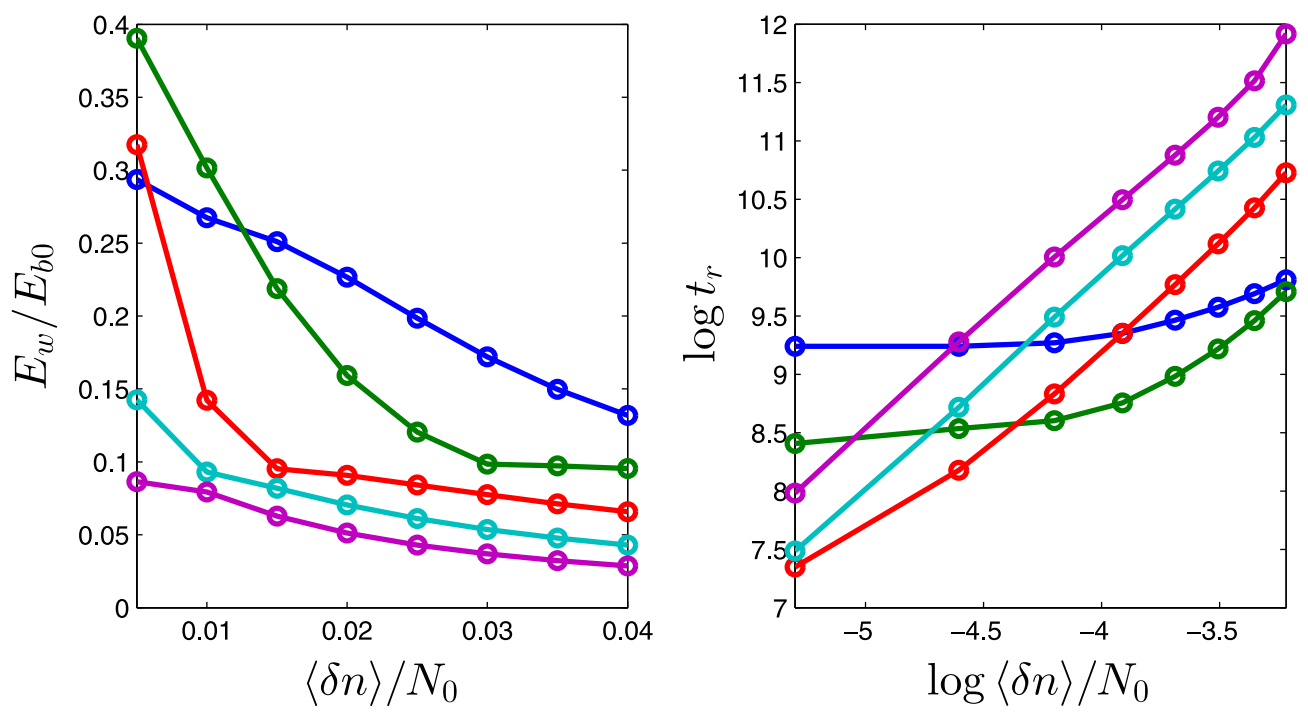

Figure 5. The maximum of the total energy of the waves, $E_{\mathrm{w}}$, (left panel) reached in the relaxation process as a function of the density fluctuation level; $E_{\mathrm{b} 0}$ is the initial energy of the beam. The different colors correspond to the different velocities of the beams (the same as in Figure 4). Right panel: the time of growth, $t_{\mathrm{r}}$, normalized to $1 / \omega_{\mathrm{p}}$ as a function of $\langle\delta n\rangle / N_{0}$ on the $\log -\log$ scale. The colors correspond to different $v_{\mathrm{b}}$. The beam density is $n_{\mathrm{b}} / N_{0}=2.5 \times 10^{-5}$.

energy. The results are consistent with values for energy transferred to beam accelerated particles, as estimated above. Thus, density fluctuations can result in an "inverse" energy flux. Energy can be transferred from "slow" electrons to "fast" electrons through the generation and absorption of Langmuir waves.

To estimate the characteristic time of wave energy growth, $t_{\mathrm{r}}$, we consider the difference in time between the moment when the total wave energy reaches $1 \%$ of the initial energy of the beam and another moment in time when the wave energy for different levels of fluctuations reaches some energy level that is the same for all simulation sets that we choose to be equal to $\min \left(E_{\max }(\langle\delta n\rangle)\right)$. We define this $\min \left(E_{\max }(\langle\delta n\rangle)\right)$ as the smallest level of all of the maximum energies of waves achieved in the set of simulations with the same $v_{\mathrm{b}}$ and different levels of fluctuation (for example, see the right panel of Figure 3). The right panel of Figure 5 displays $t_{\mathrm{r}}$ as a function of $\langle\delta n\rangle / N_{0}$ for different values of the beam velocity. As can be seen from the figure, for cases with $(1 / 2)\left(\langle\delta n\rangle / N_{0}\right) \leqslant v_{\mathrm{t}}^{2} / v_{\mathrm{b}}^{2}$ (the blue line in Figure 5), an increase in the fluctuation level results in a slight increase in the characteristic time of growth. However, for cases with $(1 / 2)\left(\langle\delta n\rangle / N_{0}\right) \geqslant v_{\mathrm{t}}^{2} / v_{\mathrm{b}}^{2}, t_{\mathrm{r}}$ increases significantly with an increase in $\langle\delta n\rangle / N_{0}$. When fluctuations strongly affect the relaxation process, $(1 / 2)\left(\langle\delta n\rangle / N_{0}\right) \gg v_{\mathrm{t}}^{2} / v_{\mathrm{b}}^{2}$, a simple approximation for $t_{r}(\langle\delta n\rangle)$ can be found, as follows: $t_{r}(\langle\delta n\rangle) \sim\langle\delta n\rangle^{-2}$.

All of the results provided above were obtained for the same value of the velocity dispersion of the beam, $\Delta v_{\mathrm{b}} / v_{\mathrm{t}}=0.5$. Now let us consider the role of the initial thermal velocity of the beam. The left panel of Figure 6 provides a ratio of the energy of the accelerated particles to the initial energy of the beam for different initial beam velocities and different initial thermal velocities of the beam particles. The energy of the accelerated particles displays the typical behavior: an increase in the density fluctuation level or the initial velocity of the beam, resulting in an increase in the energy of accelerated particles. However, an increase in the initial thermal velocity of beam electrons leads to a decrease in $E_{\mathrm{a}}$. For instance, for the case with $v_{\mathrm{b}}=10 v_{\mathrm{t}}$ and an initial thermal velocity of
$\Delta v_{\mathrm{b}}=0.5 v_{\mathrm{t}}$ (the red solid line in Figure 6), the ratio $E_{\mathrm{a}} / E_{\mathrm{b} 0}$ at the end of the relaxation is equal to $58 \%$ for $\langle\delta n\rangle / N_{0}=0.04$. An increase in the beam thermal velocity to $v_{\mathrm{t}}$ results in a decrease of $E_{\mathrm{a}} / E_{\mathrm{b} 0}$ to $43 \%$ (the red dashed line in Figure 6). For a beam with the same $v_{\mathrm{b}}$ and for the thermal velocity, $\Delta v_{\mathrm{b}}=2 v_{\mathrm{t}}$, the energy of accelerated particles decreases to a value of only $15 \%$ from the initial energy of the beam (the red dotted line in Figure 6). The result is expected because we defined the accelerated particles as particles with velocities larger than $v_{\mathrm{b}}+3 \Delta v_{\mathrm{b}}$. According to this definition, for beams with $v_{\mathrm{b}}=14 v_{\mathrm{t}}$ and $\Delta v_{\mathrm{b}}=0.5 v_{\mathrm{t}}$, only particles with velocities larger than $15.5 v_{\mathrm{t}}$ may be considered as accelerated. At the same time, for a case with $v_{\mathrm{b}}=14 v_{\mathrm{t}}$ and $\Delta v_{\mathrm{b}}=2 v_{\mathrm{t}}$, electrons considered as accelerated should have velocities larger than $20 v_{\mathrm{t}}$. For both cases, the energy of particles with velocities larger than $18 v_{\mathrm{t}}$ differs slightly.

The right panel of Figure 6 provides the maximum for total wave energy reached during the relaxation process as a function of the level of density fluctuations for beams with a different initial $v_{\mathrm{b}}$ and a different thermal velocity $\Delta v_{\mathrm{b}}$. The growth rate of waves depends on the derivative of the distribution function. Thus, a change in the initial thermal velocity of the beam should cause changes in the region of the phase space where waves can grow effectively. On the other hand, the presence of density fluctuations results in a broadening of the resonant conditions of the wave-particle interactions and a modification of the region where waves can be generated. As a result, two parameters that determine the efficiency of wave growth during relaxation (for the same initial velocity of the beam) are provided. The red lines in Figure 6 correspond to relaxation of a beam with $v_{\mathrm{b}}=10 v_{\mathrm{t}}$. As can be seen, while $(1 / 2)\left(\langle\delta n\rangle / N_{0}\right) \leqslant v_{\mathrm{t}}^{2} / v_{\mathrm{b}}^{2}\left(\langle\delta n\rangle / N_{0} \leqslant 0.025\right.$ for the present beam), changes in the initial thermal velocity of the beam lead to significant changes in the rate of wave generation. For instance, for a beam with $\Delta v_{\mathrm{b}}=2 v_{\mathrm{t}}$ (the red dotted line in Figure 6) and $\langle\delta n\rangle / N_{0}=0.02$, the maximum for total wave energy is equal to $15 \%$ of the initial energy of the beam. A decrease of $\Delta v_{\mathrm{b}}$ to $0.5 v_{\mathrm{t}}$ results in a decrease in $E_{\mathrm{w}}$ to $10 \%$. However, an increase in the density fluctuation level 

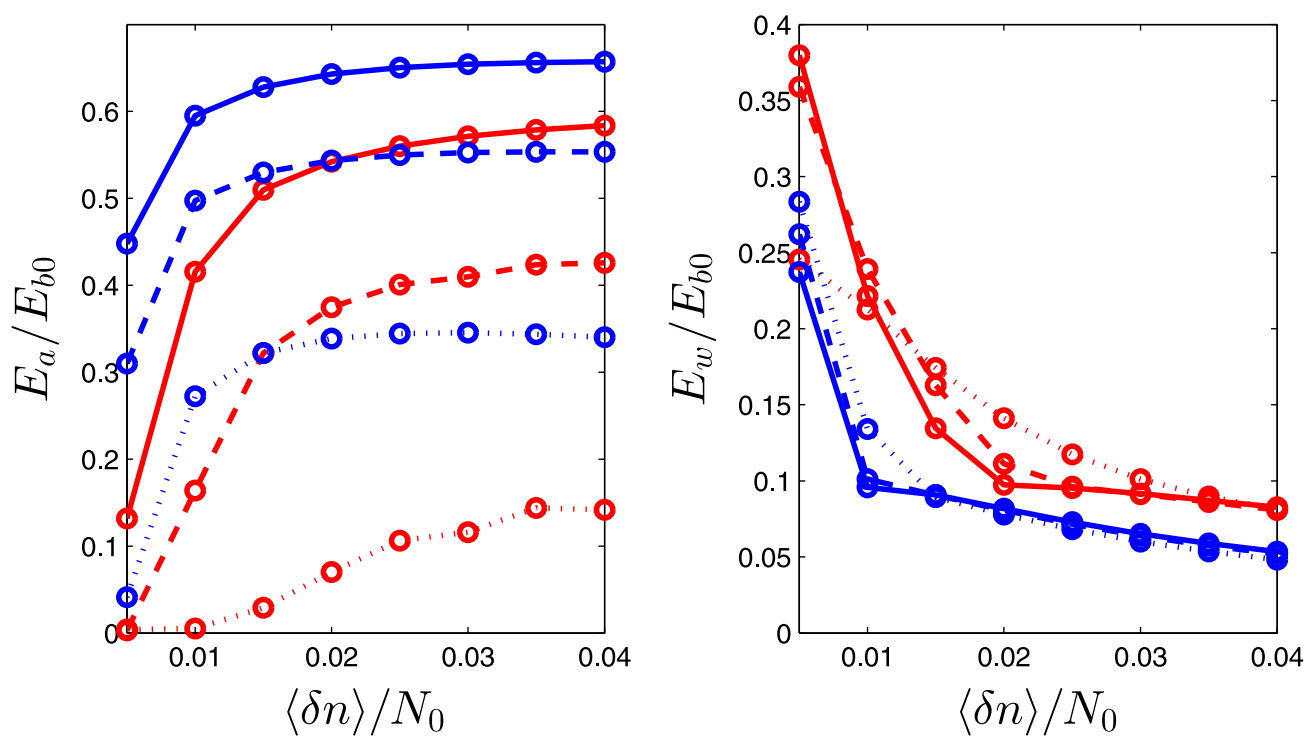

Figure 6. The energy of the accelerated particles, $E_{\mathrm{a}}$, (left panel) and maximum of the total energy of the waves, $E_{\mathrm{w}}$, (right panel) reached during relaxation as a function of the level of the density fluctuations. The colors correspond to the different initial velocities of the beam, as follows: red color, $v_{\mathrm{b}}=10 v_{\mathrm{t}}$; and blue color, $v_{\mathrm{b}}=14 v_{\mathrm{t}}$. The line styles correspond to the different initial thermal velocities of the beam, as follows: solid line, $\Delta v_{\mathrm{b}}=0.5 v_{\mathrm{t}}$; dashed line, $\Delta v_{\mathrm{b}}=1 v_{\mathrm{t}}$; and dotted line, $\Delta v_{\mathrm{b}}=2 v_{\mathrm{t}}$.

reduces the role of the initial thermal dispersion of the beam. For a beam with $v_{\mathrm{b}}=14 v_{\mathrm{t}}$ (the blue lines in Figure 6), beginning with a level of density fluctuation equal to 0.015 , there is no significant difference in the $E_{\mathrm{w}}$ for cases with a different initial $\Delta v_{\mathrm{b}}$. Thus, for fluctuation levels corresponding to condition $(1 / 2)\left(\langle\delta n\rangle / N_{0}\right) \gg v_{\mathrm{t}}^{2} / v_{\mathrm{b}}^{2}$, resonance broadening plays a major role in the process of wave generation.

In a homogeneous plasma, the time of wave growth can be written in the following form:

$$
t_{r}=\frac{W_{\text {end }}}{W_{\text {init }}} \frac{1}{\omega_{\mathrm{p} 0}} \frac{N_{0}}{n_{\mathrm{b}}} \frac{\Delta v_{\mathrm{b}}^{2}}{v_{\mathrm{b}}^{2}}
$$

where $W_{\text {end }}$ and $W_{\text {init }}$ are the energies of the waves at the end and beginning, respectively, of the relaxation process. One can note that an increase in the initial thermal velocity of the beam should lead to an increase in the time of growth. For two beams with thermal velocities $\Delta v_{\mathrm{b} 1}$ and $\Delta v_{\mathrm{b} 2}$ and the same $v_{\mathrm{b}}$, the ratio of $t_{\mathrm{r} 1}$ and $t_{\mathrm{r} 2}$ is as follows:

$$
\frac{t_{\mathrm{r} 1}}{t_{\mathrm{r} 2}}=\left(\frac{\Delta v_{\mathrm{b} 1}}{\Delta v_{\mathrm{b} 2}}\right)^{2} .
$$

Figure 7 provides the ratio of the times of wave growth for beams with different $v_{\mathrm{b}}$ and $\Delta v_{\mathrm{b}}$ as a function of the level of density fluctuations. As can be seen, when the level of fluctuations is high enough $\left((1 / 2)\left(\langle\delta n\rangle / N_{0}\right) \gg v_{\mathrm{t}}^{2} / v_{\mathrm{b}}^{2}\right)$, the change in the initial velocity of the beam does not strongly affect the time of wave growth.

\section{SUMMARY AND DISCUSSION}

Here we propose a statistical model that describes the relaxation of an electron beam in a plasma with a relatively small scale $\left(10^{2} \lambda_{\mathrm{D}}-10^{5} \lambda_{\mathrm{D}}\right)$ of density fluctuations. Using the model, we describe the evolution of the electron distribution function and the energy of Langmuir waves. The suggested one-dimensional (1D) numerical scheme is applicable for

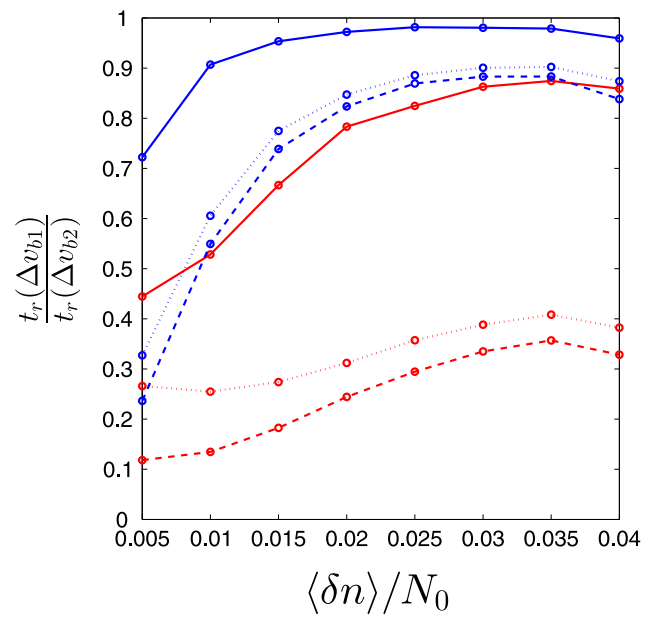

Figure 7. The ratio of times $t_{\mathrm{r}}$ for various thermal velocities of the beam as a function of the level of density fluctuations. The colors correspond to the various initial velocities of the beam, as follows: red color, $v_{\mathrm{b}}=10 v_{\mathrm{t}}$; and blue color, $v_{\mathrm{b}}=14 v_{\mathrm{t}}$. The line styles correspond to the various initial thermal velocities of the beam, as follows: solid line, $t_{r}\left(\Delta v_{\mathrm{b}}=0.5 v_{\mathrm{t}}\right) / t_{r}\left(\Delta v_{\mathrm{b}}=1 v_{\mathrm{t}}\right)$; dashed line, $t_{r}\left(\Delta v_{\mathrm{b}}=0.5 v_{\mathrm{t}}\right) / t_{r}\left(\Delta v_{\mathrm{b}}=2 v_{\mathrm{t}}\right) ; \quad$ and dotted line, $t_{r}\left(\Delta v_{\mathrm{b}}=1 v_{\mathrm{t}}\right) / t_{r}\left(\Delta v_{\mathrm{b}}=2 v_{\mathrm{t}}\right)$.

physical parameters of the solar wind plasma at different distances from the Sun (e.g., $\sim 1 \mathrm{AU})$. Thus, we can use our computations to describe type III solar bursts, as well as beam plasma interactions, within the vicinity of the heliosphere and planetary shocks.

Using the model, the computations require much less computer resources than particle-in-cell simulations or the Zakharov technique. However, the proposed numerical scheme is $1 \mathrm{D}$ and, thus, the effects of the angular diffusion of Langmuir waves on density fluctuations were ignored. At this stage the model does not include the effects of collision losses, ionsound waves, and the generation of electromagnetic emissions. For this reason, the system conserves the total energy that can be transferred from the beam to the waves and backward during the relaxation process. The relationships between energy or the 
number of accelerated particles and the levels of the density fluctuations, $n_{\mathrm{a}} / n_{\mathrm{b}}, E_{\mathrm{a}} / E_{\mathrm{b} 0} \sim \sqrt{\langle\delta n\rangle / N_{0}}$, are quite simple for cases with $(1 / 2)\left(\langle\delta n\rangle / N_{0}\right) \gg v_{\mathrm{t}}^{2} / v_{\mathrm{b}}^{2}$. The goal of the present work is to show the importance of small-scale density fluctuations and to propose a self-consistent and closed description of the beam relaxation process.

In the study, numerical simulations indicated that there are three key parameters that influence the relaxation process: the ratio of the initial beam velocity $v_{\mathrm{b}}$ to the thermal velocity $v_{\mathrm{t}}$ of a background plasma, the level of the density fluctuations $\langle\delta n\rangle / N_{0}$, and the width of the beam in velocity space. Depending on the values of $v_{\mathrm{b}}^{2} / v_{\mathrm{t}}^{2}$ and $\langle\delta n\rangle / N_{0}$, three different scenarios for relaxation can be distinguished. (1) $(1 / 2)\left(\langle\delta n\rangle / N_{0}\right) \ll v_{\mathrm{t}}^{2} / v_{\mathrm{b}}^{2}$, where the wave excitation process is very similar to the one taking place in the homogeneous plasma. Relaxation results in plateau formation of the electron distribution function without a significant increase in the population of energetic particles. The total energy of waves at the end of the relaxation equals $30 \%$ of the initial energy of the beam, which is in good agreement with predictions of $\mathrm{QL}$ theory (Vedenov et al. 1962). (2) $(1 / 2)\left(\langle\delta n\rangle / N_{0}\right) \sim v_{\mathrm{t}}^{2} / v_{\mathrm{b}}^{2}$ corresponds to the intermediate regime. Density fluctuations can affect the phase velocity of waves. Thus, reabsorption of a portion of the wave energy by energetic electrons occurs, but the number of accelerated particles is still relatively low. (3) Under the condition $(1 / 2)\left(\langle\delta n\rangle / N_{0}\right) \gg v_{\mathrm{t}}^{2} / v_{\mathrm{b}}^{2}$ the process of beam relaxation is totally determined by density fluctuations. The resonance broadening caused by density fluctuations allows the wave to interact with particles over a wide range of velocities. There is a very efficient energy transfer from electrons with velocities of $v<v_{\mathrm{b}}$ to higher energy electrons with $v>v_{\mathrm{b}}$ via the generation and reabsorption of Langmuir waves. The case is characterized by a low level of total wave energy at the end of the relaxation process. In contrast, the energy of accelerated electrons can reach up to $70 \%$ from the initial energy of the beam for cases with a small initial spread for a beam in the velocity space. However, as in a homogeneous plasma, relaxation runs mainly toward low velocities and finishes with the formation of a plateau.

An increase in the density fluctuation level results in an increase in the time characterizing wave energy growth, $t_{\mathrm{r}}$. The average growth rate of the waves, $\gamma$, and the diffusion coefficient, $D$, strongly depend on the magnitude of the fluctuations. High amplitude fluctuations can strongly impact the resonant condition of the wave-particle interaction, which leads to broadening of the resonance in the phase space and the wave damping. This, in turn, significantly slows down the relaxation. In the case of the Gaussian distribution for the fluctuation magnitudes and for a high level of density fluctuations, a simple relationship between $t_{\mathrm{r}}$ and $\langle\delta n\rangle$ was found, as follows: $t_{r}(\langle\delta n\rangle) \sim\langle\delta n\rangle^{-2}$.

V.K. acknowledges the financial support of the Centre National d'Etudes Spatiales (CNES) through the grant "Invited scientist STEREO S/WAVES." A.V. and V.K. are grateful to Dr. V. Olkhov for useful discussions.

\section{APPENDIX A EQUATION FOR THE WAVE}

Here, we begin with a consideration of the interaction of a single electron with a monochromatic wave. In a $1 D$ case the equation of motion can be written as follows:

$$
\frac{d v}{d t}=-\frac{e E}{m} \cos (k x-\omega t+\tilde{\phi}),
$$

where $v$ is the electron velocity, $E$ is the wave amplitude, and $\tilde{\phi}$ is the initial phase of the wave.

Using perturbation theory for the velocity, $v=v_{0}+v_{1}+v_{2}$, where $v_{0}$ is the unperturbed velocity of the particle, one can write equations for the first two terms in the form of a power series, as follows:

$$
\frac{d v_{1}}{d t}=-\frac{e E}{m} \cos \left(\left(k v_{0}-\omega\right) t+\phi\right)
$$

and

$$
\begin{aligned}
\frac{d v_{2}}{d t}= & -\frac{e E}{m}\left[\cos \left(\left(k v_{0}-\omega\right) t+k \int_{0}^{t} v_{1} d t+\phi\right)\right. \\
& \left.-\cos \left(\left(k v_{0}-\omega\right) t+\phi\right)\right],
\end{aligned}
$$

where $\phi=\tilde{\phi}+k x_{0}$.

Taking into account that $\cos \left(k \int_{0}^{t} v_{1} d t\right) \approx 1$ and $\sin \left(k \int_{0}^{t} v_{1} d t\right) \approx k \int_{0}^{t} v_{1} d t$, one can rewrite the second term as follows:

$$
\frac{d v_{2}}{d t}=\frac{e E}{m} k \int_{0}^{t} v_{1} d t \sin \left(\left(k v_{0}-\omega\right) t+\phi\right) .
$$

Following integration in Equation (8) over a small time interval $t \rightarrow 0$, the first term can be rewritten as follows:

$$
\begin{aligned}
v_{1}= & -\frac{e E}{m} \frac{1}{\left(k v_{0}-\omega\right)} \\
& \times\left[\sin \left(\left(k v_{0}-\omega\right) t+\phi\right)-\sin (\phi)\right] .
\end{aligned}
$$

Now, it is easy to find

$$
\begin{aligned}
\int_{0}^{t} v_{1} d t= & \frac{e E}{m} \frac{1}{\left(k v_{0}-\omega\right)^{2}} \\
& \times\left[\cos \left(\left(k v_{0}-\omega\right) t+\phi\right)-\cos (\phi)\right] \\
& +\frac{e E}{m} \frac{t}{\left(k v_{0}-\omega\right)} \sin (\phi) .
\end{aligned}
$$

Following substitution of $\int_{0}^{t} v_{1} d t$ in Equation (9) one can obtain

$$
\begin{aligned}
\frac{d v_{2}}{d t}= & \frac{e^{2} E^{2}}{m^{2}} \frac{k}{\left(k v_{0}-\omega\right)^{2}}\left[\cos \left(\left(k v_{0}-\omega\right) t+\phi\right)\right. \\
& -\cos (\phi)] \sin \left(\left(k v_{0}-\omega\right) t+\phi\right) \\
& +\frac{e^{2} E^{2}}{m^{2}} \frac{k t}{\left(k v_{0}-\omega\right)} \sin (\phi) \\
& \times \sin \left(\left(k v_{0}-\omega\right) t+\phi\right) .
\end{aligned}
$$

By keeping the terms to a second order of accuracy, the equation for changes in the electron energy can be written as follows:

$$
\frac{d \epsilon}{d t}=m v_{1} \frac{d v_{1}}{d t}+m v_{0} \frac{d v_{2}}{d t}
$$


Substituting $v_{1}, d v_{1} / d t$, and $d v_{2} / d t$ into (12) one can find

$$
\begin{aligned}
\frac{d \epsilon}{d t}= & \frac{e^{2} E^{2}}{m} \frac{1}{\left(k v_{0}-\omega\right)}\left[\sin \left(\left(k v_{0}-\omega\right) t+\phi\right)\right. \\
& -\sin (\phi)] \cos \left(\left(k v_{0}-\omega\right) t+\phi\right) \\
& +\frac{e^{2} E^{2}}{m} \frac{v k}{\left(k v_{0}-\omega\right)^{2}}\left[\cos \left(\left(k v_{0}-\omega\right) t+\phi\right)\right. \\
& -\cos (\phi)] \sin \left(\left(k v_{0}-\omega\right) t+\phi\right) \\
& +\frac{e^{2} E^{2}}{m} \frac{v k t}{\left(k v_{0}-\omega\right)} \sin (\phi) \sin \left(\left(k v_{0}-\omega\right) t+\phi\right) .
\end{aligned}
$$

By taking into account that $\phi$ is a random variable with a uniform distribution, it is necessary to average (13) over $\phi$, as follows:

$$
\begin{aligned}
\left\langle\frac{d \epsilon}{d t}\right\rangle_{\phi}= & \frac{e^{2} E^{2}}{2 m^{2}} \frac{1}{\left(k v_{0}-\omega\right)} \sin \left(\left(k v_{0}-\omega\right) t\right) \\
& -\frac{e^{2} E^{2}}{2 m^{2}} \frac{v_{0} k}{\left(k v_{0}-\omega\right)^{2}} \sin \left(\left(k v_{0}-\omega\right) t\right) \\
& +\frac{e^{2} E^{2}}{2 m^{2}} \frac{v k t}{\left(k v_{0}-\omega\right)} \cos \left(\left(k v_{0}-\omega\right) t\right) .
\end{aligned}
$$

Due to fluctuations of the plasma density, the phase velocity of the wave can have various values at different intervals. With a probability distribution function $P(V)$, one can average Equation (14) over $V$, as follows:

$$
\begin{aligned}
\left\langle\frac{d \epsilon}{d t}\right\rangle_{\phi V}= & \frac{e^{2} E^{2}}{2 m} \int_{0}^{\infty}\left[\frac{1}{k(v-V)} \sin (k(v-V) t)\right. \\
& -\frac{v}{k(v-V)^{2}} \sin (k(v-V) t) \\
& \left.+\frac{v t}{(v-V)} \cos (k(v-V) t)\right] P(V) d V
\end{aligned}
$$

Here we consider the particle ensemble with a given distribution function, $f(v)$. The change in the energy of a wave over time $t$ is equal to the total change of the particle energy at a volume taken with an opposite sign, as follows:

$$
\frac{d W}{d t}=-n_{\mathrm{b}} \int_{-\infty}^{\infty}\left\langle\frac{d \epsilon}{d t}\right\rangle_{\phi V} f(v) d v
$$

Changing the order of the integration in (16) and making a Taylor series expansion for $f(v)$, Equation (16) can be rewritten as follows:

$$
\begin{aligned}
\frac{d W}{d t}= & \frac{e^{2} E^{2}}{2 m} n_{\mathrm{b}} \int_{0}^{\infty}\left[\frac{V^{2}}{\omega} f(V) \int_{-\infty}^{\infty} \frac{\sin ((v-V) k t)}{(v-V)^{2}} d v\right. \\
& \left.+\left.\frac{V^{2}}{\omega} \frac{\partial f(v)}{\partial v}\right|_{v=V} \int_{-\infty}^{\infty} \frac{\sin (k(v-V) t)}{v-V} d v\right] \\
& \times P_{\omega}(V) d V .
\end{aligned}
$$

The remaining terms in the expansion are proportional to $t$ and do not make any contribution to the integrals. The first term in Equation (17) is equal to zero because the integrand is an odd function. After simple calculations, the second term can be written as $\int_{-\infty}^{\infty}(\sin (x) / x) d x=\pi$. By taking into account the fact that $W=\pi E^{2} / 8$ and $\omega_{\mathrm{p} 0}^{2}=4 \pi e^{2} N_{0} / m$, the following equation can be obtained:

$$
\begin{gathered}
\frac{d W}{d t}=\frac{\omega_{\mathrm{p} 0}}{\pi} \frac{n_{\mathrm{b}}}{N_{0}} W \int_{0}^{\infty} V^{2} \frac{\partial f}{\partial V} P_{\omega}(V) d V . \\
\text { APPENDIX B } \\
\text { THE EQUATION FOR THE ELECTRON } \\
\text { DISTRIBUTION FUNCTION }
\end{gathered}
$$

Here, we introduce a probability density function $U\left(v, t \mid v_{0}, t_{0}\right)$ for the electron velocity. The function provides the probability that a particle with a velocity $v_{0}$ in the moment of time $t_{0}$ will have a velocity $v$ at time $t$ and satisfies the following Fokker-Planck equation:

$$
\begin{aligned}
\frac{\partial U\left(v, t \mid v_{0}, t_{0}\right)}{\partial t}= & -\frac{\partial}{\partial v} A(v) U\left(v, t \mid v_{0}, t_{0}\right) \\
& +\frac{\partial^{2}}{\partial v^{2}} B(v) U\left(v, t \mid v_{0}, t_{0}\right)
\end{aligned}
$$

Here, functions $A(V)$ and $B(v)$ represent the averaged characteristics for variations in the velocity and its dispersion, as follows:

$$
\begin{gathered}
A(v)=\frac{\left\langle v_{2}\right\rangle_{\phi V}}{t}, \\
B(v)=\frac{1}{2} \frac{\left\langle v_{1}^{2}\right\rangle_{\phi V}}{t},
\end{gathered}
$$

where the brackets indicate averaging over $\phi$ and $V$.

To define an equation for the electron distribution function, it is necessary to multiply Equation (19) by $f\left(v_{0}, t_{0}\right)$ and integrate it over $v_{0}$, as follows:

$$
\frac{\partial f(v, t)}{\partial t}=-\frac{\partial}{\partial v} A(v) f(v, t)+\frac{\partial^{2}}{\partial v^{2}} B(v) f(v, t) .
$$

Subsequent to integration in Equation (9), one can write $v_{2}$ as follows:

$$
\begin{aligned}
v_{2}(v, \phi)= & \frac{e^{2} E^{2}}{2 m^{2}} \frac{k}{\left(k v_{0}-\omega\right)^{3}}\left(\sin ^{2}\left(\left(k v_{0}-\omega\right) t+\phi\right)\right. \\
& -\sin ^{2}(\phi)+2 \cos (\phi) \cos \left(\left(k v_{0}-\omega\right) t+\phi\right) \\
& \left.+2 \sin (\phi) \sin \left(\left(k v_{0}-\omega\right) t+\phi\right)-2\right) \\
& -\frac{e^{2} E^{2}}{m^{2}} \frac{k t}{\left(k v_{0}-\omega\right)^{2}} \sin (\phi) \cos \left(\left(k v_{0}-\omega\right) t+\phi\right) .
\end{aligned}
$$

Now it is easy to determine $\left\langle v_{1}^{2}\right\rangle_{\phi}$ and $\left\langle v_{2}\right\rangle_{\phi}$, as follows:

$$
\begin{aligned}
\left\langle v_{2}\right\rangle_{\phi}= & \frac{e^{2} E^{2}}{m^{2}} \frac{k}{\left(k v_{0}-\omega\right)^{3}}\left(\cos \left(\left(k v_{0}-\omega\right) t\right)-1\right) \\
& +\frac{e^{2} E^{2}}{2 m^{2}} \frac{k t}{\left(k v_{0}-\omega\right)^{2}} \sin \left(\left(k v_{0}-\omega\right) t\right),
\end{aligned}
$$




$$
\left\langle v_{1}^{2}\right\rangle_{\phi}=\frac{e^{2} E^{2}}{m^{2}} \frac{1}{(k v-\omega)^{2}}(1-\cos ((k v-\omega) t)) .
$$

By taking into account that $t=a / v, A(v)$ and $B(v)$ can be written as follows:

$$
\begin{gathered}
A(v)=\frac{e^{2} E^{2}}{m^{2}} \int_{0}^{\infty}\left[\frac{v}{a k^{2}(v-V)^{3}}(\cos ((v-V) k t)-1)\right. \\
\left.+\frac{1}{2 k(v-V)^{2}} \sin ((v-V) k t)\right] P_{\omega}(V) d V \\
B(v)=\frac{e^{2} E^{2}}{2 m^{2}} \int_{0}^{\infty} \frac{v}{a k^{2}(v-V)^{2}} \\
\quad \times(1-\cos ((v-V) k t)) P_{\omega}(V) d V .
\end{gathered}
$$

Here, it is also worth writing the following equation for $\partial B(v) / \partial v$ :

$$
\begin{aligned}
\frac{\partial B(v)}{\partial v}= & \frac{e^{2} E^{2}}{2 m^{2}} \int_{0}^{\infty}\left(\frac{V+v}{a k^{2}(v-V)^{3}}(\cos ((v-V) k t)-1)\right. \\
& \left.+\frac{V}{k v(v-V)^{2}} \sin ((v-V) k t)\right) P_{\omega}(V) d V .
\end{aligned}
$$

By taking the first derivative of the second term of Equation (20) and substituting $A(v), B(v)$, and $\partial B(v) / \partial v$, one can obtain an equation for the electron distribution function in the following form:

$$
\begin{aligned}
\frac{\partial f}{\partial t}= & -\frac{e^{2} E^{2}}{2 m^{2}} \frac{\partial}{\partial v}\left[\int_{0}^{\infty} \frac{1}{a k^{2}(v-V)^{2}}\right. \\
& \times(\cos ((v-V) k t)-1) P_{\omega}(V) d V f(v) \\
& +\int_{0}^{\infty} \frac{1}{k v(v-V)} \sin ((v-V) k t) P_{\omega}(V) d V f(v) \\
& -\int_{0}^{\infty} \frac{1}{t k^{2}(v-V)^{2}}(1-\cos ((v-V) k t)) \\
& \left.\times P_{\omega}(V) d V \frac{\partial f}{\partial v}\right] .
\end{aligned}
$$

Through a Taylor series expansion of $P(V)$ at $V=v$, one can integrate the terms in Equation (21), as follows:

$$
\begin{aligned}
& \int_{0}^{\infty} \frac{1}{a k^{2}(v-V)^{2}}(\cos ((V-v) k t)-1) P_{\omega}(V) d V f(v) \\
&=-P_{\omega}(v) \frac{2}{\omega} \int_{0}^{\infty} \frac{1}{k t(V-v)^{2}} \sin ^{2}\left((V-v) \frac{k t}{2}\right) d V f(v) \\
&=-P_{\omega}(v) \frac{\pi}{2 \omega} f(v) \\
& \int_{0}^{\infty} \frac{1}{k v(v-V)} \sin ((v-V) k t) P_{\omega}(V) d V f(v) \\
&=P_{\omega}(v) \frac{1}{\omega} \int_{0}^{\infty} \frac{1}{(V-v)} \sin ((V-v) k t) d V f(v) \\
&=P_{\omega}(v) \frac{\pi}{2 \omega} f(v)
\end{aligned}
$$

$$
\begin{aligned}
\int_{0}^{\infty} & \frac{1}{t k^{2}(v-V)^{2}}(1-\cos ((v-V) k t)) P_{\omega}(V) d V \frac{\partial f}{\partial v} \\
= & P_{\omega}(v) \frac{2 v}{\omega} \int_{0}^{\infty} \frac{1}{t k(V-v)^{2}} \sin ^{2}\left((V-v) \frac{k t}{2}\right) d V \frac{\partial f}{\partial v} \\
= & \frac{\pi}{2} \frac{v}{\omega} P_{\omega}(v) \frac{\partial f}{\partial v} .
\end{aligned}
$$

After substitution of the results into Equation (21), one can find the final equation for the $f(v, t)$ function, as follows:

$$
\frac{\partial f}{\partial t}=\frac{2 e^{2} W}{m^{2} \omega} \frac{\partial}{\partial v} v P_{\omega}(v) \frac{\partial f}{\partial v} .
$$

\section{APPENDIX C CONSERVATION LAW}

Equations (18) and (22) constitute the basic system of equations for our model. To make this system more convenient for numerical simulation, here we introduce the following dimensionless variables: $\tilde{t}=\left(\pi / \omega_{\mathrm{p} 0}\right)\left(N_{0} / n_{\mathrm{b}}\right) t, \quad \tilde{v}=v_{\mathrm{t}} v$, $\tilde{f}=f / v_{\mathrm{t}}, \tilde{P}_{\omega}(V)=P_{\omega}(V) / v_{\mathrm{t}}$, and $\tilde{W}=2 \pi^{2} n_{\mathrm{b}} m v_{\mathrm{t}}^{2} W$. We omit tildes to simply the form of the equations. The system takes the following form:

$$
\begin{gathered}
\frac{\partial f}{\partial t}=\frac{\partial}{\partial v} v W P_{\omega}(v) \frac{\partial f}{\partial v}, \\
\frac{d W}{d t}=\int_{0}^{\infty} W V^{2} \frac{\partial f}{\partial V} P_{\omega}(V) d V .
\end{gathered}
$$

One can easily determine that the system has a conservation law for total energy, as follows:

$$
\begin{aligned}
\frac{\partial}{\partial t}\left[\frac{1}{2} \int_{0}^{\infty} v^{2} f(v) d v+W\right]= & \frac{1}{2} \int_{0}^{\infty} v^{2} \frac{\partial f(v)}{\partial t} d v+\frac{\partial W}{\partial t} \\
= & \frac{1}{2} \int_{0}^{\infty} v^{2} \frac{\partial}{\partial v} v W P_{\omega}(v) \frac{\partial f}{\partial v} d v \\
& +\int_{0}^{\infty} W V^{2} \frac{\partial f}{\partial V} P_{\omega}(V) d V \\
= & -\int_{0}^{\infty} W v^{2} P_{\omega}(v) \frac{\partial f}{\partial v} d v \\
& +\int_{0}^{\infty} W V^{2} \frac{\partial f}{\partial V} P_{\omega}(V) d V \\
= & 0 .
\end{aligned}
$$

\section{REFERENCES}

\footnotetext{
Austin, D. R., Hole, M. J., Robinson, P. A., Cairns, I. H., \& Dallaqua, R. 2007, PhRvL, 99, 205004

Bale, S. D., Larson, D. E., Lin, R. P., et al. 2000, JGR, 105, 27353

Breizman, B. N., \& Ruytov, D. D. 1969, JETP, 30, 759

Buttighoffer, A. 1998, A\&A, 335, 295

Cairns, I. H. 1988, JGR, 93, 3958

Cairns, I. H., \& Grubits, K. A. 2001, PhRvE, 64, 056408

Cairns, I. H., \& Melrose, D. B. 1985, JGR, 90, 6637

Cairns, I. H., \& Menietti, J. D. 1999, JGR, 106, 29515

Cairns, I. H., \& Robinson, P. A. 1999, PhRvL, 82, 3066

Celnikier, L. M., Harvey, C. C., Jegou, R., Moricet, P., \& Kemp, M. 1983, A\&A, 126, 293
}

Celnikier, L. M., Muschietti, L., \& Goldman, M. V. 1987, A\&A, 181, 138 
Chen, C. H. K., Salem, C. S., Bonnell, J. W., Mozer, F. S., \& Bale, S. D. 2012, PhRvL, 109, 035001

Drummond, W. E., \& Rosenbluth, M. N. 1962, PhFl, 5, 1507

Dulk, G. A. 1985, ARA\&A, 23, 169

Emslie, A. G., Dennis, B. R., Shih, A. Y., et al. 2012, ApJ, 759, 71

Ergun, R. E., Larson, D., Lin, R. P., et al. 1998, ApJ, 503, 435

Ergun, R. E., Malaspina, R. E., Cairns, I. H., et al. 2008, PhRvL, 101, 051101

Forman, M. A., Wicks, R. T., \& Horbury, T. S. 2011, ApJ, 733, 76

Galeev, A. A., Sagdeev, R. Z., Shapiro, V. D., \& Shevchenko, V. I. 1977a, JETP, 46, 71

Galeev, A. A., Sagdeev, R. Z., Shapiro, V. D., \& Shevchenko, V. I. 1977b, JETP, 72, 506

Ginzburg, V. L., \& Zhelezniakov, V. V. 1958, SvA, 2, 653

Goldman, M. V. 1983, SoPh, 89, 403

Gurnett, D. A., Anderson, R. R., Scarf, F. L., \& Kurth, W. S. 1978, JGR, 83,4147

Hinkel-Lipsker, D. E., Fried, D. E., \& Morales, B. D. 1992, PhFlB, 4, 559

Kaplan, S. A., \& Tsytovich, V. N. 1973, Plasma Astrophysics (Oxford: Pergamon)

Kellogg, P. J. 1986, A\&A, 169, 329

Kellogg, P. J., Goetz, K., Monson, S. J., \& Bale, S. D. 1999, JGR, 104, 17069

Kellogg, P. J., \& Horbury, T. S. 2005, AnGeo, 23, 3765

Kontar, E. P. 2001, A\&A, 375, 629

Kontar, E. P., \& Reid, H. A. S. 2009, ApJL, 695, L140

Krafft, C., Volokitin, A. S., \& Krasnoselskikh, V. V. 2013, ApJ, 778, 111

Krasnoselskikh, V. V., Dudok de Wit, T., \& Bale, S. D. 2011, AnGeo, 29, 613

Krasnoselskikh, V. V., Lobzin, V. V., Musatenko, K., et al. 2007, JGRA, 112, 10109

Krucker, S., Oakley, P. H., \& Lin, R. P. 2009, ApJ, 691, 806

Li, B., Robinson, P. A., \& Cairns, I. H. 2006, PhPl, 13, 082305

Lin, R. P., Potter, D. W., Gurnett, D. A., \& Scarf, F. L. 1981, ApJ, 251, 364

Malaspina, D. M., Cairns, I. H., \& Ergun, R. E. 2012, ApJ, 755, 45
Malaspina, D. M., \& Ergun, R. E. 2008, JGRA, 113, 12108

Malaspina, D. M., Kellogg, P. J., Bale, S. D., \& Ergun, R. E. 2010, ApJ, 711,322

Melrose, D. B. 1980, Plasma Astrophysics: Nonthermal Processes in Diffuse Magnetized Plasmas, Vol. 1 (New York: Gordon and Breach)

Melrose, D. B. 1987, SoPh, 111, 89

Melrose, D. B., Cairns, I. H., \& Dulk, G. A. 1986, A\&A, 163, 229

Muschietti, L., Goldman, M. V., \& Newman, D. 1985, SoPh, 96, 181

Neugebauer, M. 1975, JGR, 80, 998

Nishikawa, K., \& Ryutov, D. D. 1976, JPSJ, 41, 1757

Papadopoulos, K., \& Freund, H. P. 1978, GeoRL, 5, 881

Ratcliffe, H., Bian, N. H., \& Kontar, E. P. 2012, ApJ, 761, 176

Reid, H. A. S., \& Kontar, E. P. 2010, ApJ, 721, 864

Reid, H. A. S., \& Kontar, E. P. 2013, SoPh, 285, 217

Reid, H. A. S., \& Ratcliffe, H. 2014, RAA, 14, 773

Robinson, P. A. 1992, PhPl, 139, 147

Robinson, P. A. 1995, PhPl, 2, 1466

Ryutov, D. D. 1969, JETP, 30, 131

Sagdeev, R. Z., \& Galeev, A. A. 1969, Nonlinear Plasma Theory (New York: Benjamin)

Smith, D. F., \& Sime, D. 1979, ApJ, 233, 998

Sturrock, P. A. 1964, in Proc. AAS-NASA Symp. Physics of Solar Flares, ed. W. N. Hess (NASA SP-50; Washington, DC: Sci. and Tech. Info. Div.), 357

Vedenov, A. A., \& Ryutov, D. D. 1975, Reviews of Plasma Physics, Vol. 6 (New York: Consultants Bureau)

Vedenov, A. A., Velikhov, E., \& Sagdeev, R. 1962, NucFS, 2, 465

Voshchepynets, A., \& Krasnoselskikh, V. 2013, AnGeo, 31, 1379

Yoon, P. H., Wu, C. S., Vinas, A. F., et al. 1994, JGR, 99, 23481

Zakharov, V. E. 1972, JETP, 35, 908

Ziebell, L. F., Yoon, P. H., Pavan, J., \& Gaelzer, R. 2011, ApJ, 727, 16 